\title{
Could Vertical Integration Increase Innovation?
}

\author{
Chenyu Yang* \\ Simon Business School, University of Rochester
}

May 10, 2017

Click here to download the latest version

\begin{abstract}
This paper studies the effects of vertical integration on innovation in the chipset and smartphone industries. I formulate and estimate a dynamic structural model of a dominant upstream chipset maker and downstream smartphone handset makers. The two sides make dynamic investment decisions and negotiate chipset prices via Nash bargaining. Using the estimates, I simulate market outcomes should the upstream firm merge with a downstream firm. I find that the vertical merger would increase innovation rates and social welfare, driven primarily by the investment coordination of the two merged firms.
\end{abstract}

\section{Introduction}

In vertical industries, upstream and downstream innovations are often complementary. Upstream firms upgrade the core technology essential to performance enhancement, and downstream firms combine the technology with innovative designs in new consumer products. Examples of complementary innovations include traction batteries (upstream) and electric vehicles (downstream), CPU's (upstream) and personal computers (downstream) and chipsets (upstream) and smartphones (downstream). This paper studies how vertical integration affects innovation, pricing and welfare.

A large body of theoretical literature (e.g., Perry (1989); Holmström and Roberts (1998); Tirole (1999); Riordan (2008); Aghion and Holden (2011)) has examined the investment and price effects of vertical integration. Vertical integration may be pro-investment by aligning the investment incentives of the merged firms, but the impact on other downstream competitors depends on two additional factors. First, faster upstream innovation lifts the technology ceiling, allowing nonintegrated downstream firms to develop higher quality products. Second, faster innovation of

*Simon Business School, University of Rochester; chenyu.yang@simon.rochester.edu. I would like to acknowledge the generous financial support provided by Rackham Graduate School and Michigan Institute for Teaching and Research in Economics (MITRE) at the University of Michigan. I am grateful for the guidance and support from my advisers Jeremy Fox, Daniel Ackerberg, Ying Fan and Srinivasaraghavan Sriram. I benefited greatly from comments of many seminar participants. I also want to thank Chia-Yen Lee for her excellent research assistance. All errors are mine. 
the integrated downstream firm reduces both the pre-innovation and post-innovation profits of its competitors. Because the marginal value of innovation is the difference between these two profits, whether these downstream firms would innovate faster is an empirical question. The second effect is similar to the economics behind how competition affects innovation (Aghion et al. (2005)).

Vertical integration also produces the well-known efficiency trade-off between two pricing forces: reduced double marginalization allows the integrated downstream firm to charge consumers lower prices, but the integrated upstream firm has an incentive to charge higher prices to the downstream rivals (raising rivals' cost or the foreclosure effect). The two effects are likely to increase the profits of the merged firms and reduce the profits of the downstream rivals, both before and after innovation. Similar to the discussion above, how the two pricing forces affect innovation is also an empirical question.

Understanding the relative magnitudes and the interaction of the investment and price effects is crucial for policy and regulation. For example, the potential trade-off between one firm's innovation and industry-wide innovation was a key issue in the European antitrust case against Microsoft in 2004. Microsoft owned the popular proprietary operating system used on computer servers and foreclosed other server software companies. Microsoft argued in its defense that the foreclosure would increase its own innovation. The European Commission, however, believed that if Microsoft were to provide downstream rivals (server software producers) with reasonable access to its upstream technology (operating system), "the positive impact on the level of innovation in the whole industry outweighed the negative impact of the dominant undertaking's incentives to innovate." 11 The court ruled against Microsoft. In effect, the authorities believed that preventing foreclosure would increase the innovation of other downstream firms, and the resulting benefits would be greater than the potential reduction in the integrated firm's innovation.

The chipset and smartphone industries provide an interesting setting to study complementary innovations. The chipset ${ }^{2}$ includes the $\mathrm{CPU}$ of a smartphone, but may also combine the functions of the GPU, modem and other components (Yang et al. (2014)). Chipset quality determines a smartphone's key metrics, such as the computing power (CPU), network support (modem), graphic rendering (GPU) and energy efficiency. The innovations of chipsets center on improving these metrics without significantly increasing manufacturing costs (Yeap (2013)). New chipsets allow handset makers to improve other hardware. For example, to increase the screen size or display resolution, handset makers need to find additional computational power to render high definition graphics without quickly draining the battery or overheating the phone, which is only possible with more advanced chipsets (Chen et al. (2013), Phone Arena (2015)). Furthermore, chipset innovation may directly add new features to a phone. For example, Qualcomm's 805 chipsets significantly reduced the time a phone needs to be fully charged (Savov (2014)).

I use a dynamic game of investment to model the innovations of the chipset and handset makers. The main novelty is that both upstream and downstream firms are dynamic. The upstream industry

\footnotetext{
${ }^{1}$ Case T-201/04, Microsoft Corp. v. Commission, 2007 E.C.R. II-3825 (Ct. First Instance).

${ }^{2}$ Sometime a smartphone modem is also called a communication chipset. Throughout this paper a chipset always means the application processor chipset, or system-on-chip (SoC).
} 
consists of a dominant firm ("Qualcomm") and a non-strategic fringe, and the downstream firms are a finite number of oligopolistically competitive handset makers. Qualcomm invests to increase the quality of its chipsets. Downstream handset makers invest to increase the quality of their handsets, but some handset makers are constrained by Qualcomm and cannot increase their handset qualities above Qualcomm's chipset quality. Handset makers also choose the proportion of their handsets that use Qualcomm chipsets. A handset maker's sunk cost of innovation depends on the amount of its quality increase and the proportion of its handsets using Qualcomm chipsets. These decisions determine the set of products in every period. Conditional on the set of products, Qualcomm and handset makers first negotiate chipset prices via Nash bargaining. Handset makers then take the chipset prices as given and set wholesale prices. The subgame perfect equilibrium of the overall static pricing game determines the period profits. When deciding whether to innovate, upstream and downstream firms weigh the gains in the present discounted values of future profits due to innovation against the sunk costs, and the dynamic innovation decisions form a Perfect Bayesian Equilibrium (PBE).

I estimate the model using data from the US smartphone market from 2009 to 2013. The estimation procedure has three steps. First, price and quantity data of handsets allow me to estimate a static random coefficient logit model of consumer demand for smartphones. I refer to a linear combination of product characteristics, where the weights are given by the estimated demand coefficients, as the quality index of the products, and I use this index to construct the quality frontiers of Qualcomm and handset makers. Next, I recover chipset prices and other marginal costs of smartphones using equilibrium pricing conditions and data on chipset markups. The first two steps do not involve estimating the dynamic model. The estimates and the pricing equilibrium assumption imply the period profit functions of the upstream and downstream firms. In the last step, I use the estimated period profit functions and the evolution of quality frontiers of Qualcomm and handset makers to estimate the innovation cost function. To keep the computation tractable, I estimate a dynamic game among the upstream Qualcomm and three handset makers: Apple, Samsung and HTC. Consistent with data, I assume that Apple only uses its own chipsets, whereas HTC only uses Qualcomm chipsets. Samsung can adjust the proportion of its handsets using Qualcomm chipsets. Samsung and HTC are constrained by Qualcomm chipset quality, while Apple is not (it can innovate to a quality level not yet reached by Qualcomm). I use a Simulated Minimum Distance estimator to estimate the model. To ensure the existence and uniqueness of the dynamic equilibrium, I make two assumptions: (1) the dynamic game has a finite horizon, and (2) firms make investment decisions sequentially within every period. I later perform robustness checks on these assumptions.

I examine the counterfactual should Qualcomm merge with HTC, a key handset maker that primarily uses Qualcomm chipsets. The vertical integration allows Qualcomm and HTC to jointly make innovation decisions. This treatment follows the view that vertical integration facilitates the transfer of knowledge input between the merged firms (Atalay, Hortaçsu and Syverson (2014); Natividad (2014)). In the main specification, I find that the upstream Qualcomm's innovation rate, defined as the average increase of quality per period, increases $13 \%$ to $35 \%$ (95\% confidence inter- 
val), and the innovation rate of the integrated HTC increases $14 \%$ to 20\%. Moreover, Samsung's innovation rate increases $9 \%$ to $22 \%$. Apple's innovation rate increases less than $3 \%$. Consumer surplus increases $4 \%$ to $8 \%$. I decompose the effects of vertical integration into the investment effects and price effects. The investment effects dominate the price effects. In addition, although the raising rivals' cost effect increases Samsung's retail prices, the elimination of double marginalization lowers HTC prices, and the overall price effects increase the consumer surplus. The findings thus suggest that vertical integration policies should fully take into account a vertical merger's dynamic implications and in particular the investment effects, which may be much larger than the price effects. The qualitative patterns are robust across a number of alternative specifications.

The model is grounded in the theory of incomplete contract (Grossman and Hart (1986); Hart and Moore (1990)). Specifically, I assume that Qualcomm and handset makers neither contract on the outcomes of innovation ex ante nor cooperate tacitly. A complete contract or tacit cooperation between Qualcomm and HTC could effectively achieve vertical integration, but such an arrangement may be unlikely in this context. First, this industry is new, and the chipset technologies are complex and rapidly improving. Firms face many unforeseen contingencies. Month-to-month adjustment of smartphone product lines (Fan and Yang (2016)) also contributes to this difficulty. Non-HTC handset makers that use Qualcomm chipsets may also be concerned about the safety of their proprietary designs if Qualcomm and HTC have a contract that coordinates their innovative activities Allain et al. (2011)). Furthermore, the ability to design in-house chipsets seems to be a desirable goal for many handset makers. Technology commentators extol how Apple's custom-designed chipsets deliver superior performance compared with other handsets that use general-purpose chipsets from Qualcomm (Colon (2013); Bradshaw (2015); Smith (2015)). Recognizing the potential performance advantage of custom-made chipsets, handset makers either maintain (like Samsung) or are trying to start their own chipset divisions (LG, Sony and Chinese handset makers such as Xiaomi and Huawei), to varying degrees of success (Sohail (2015); Low (2017)). Formally identifying the degree of cooperation requires excluded demand shifters not available in my data. I instead use accounting investment and cost data in financial reports to provide additional evidence that the assumption of incomplete contract is appropriate. In the absence of both demand shifters and cost data, the paper provides an approach to quantify the upper bound of the benefits of vertical integration.

Related Literature and Contributions Lafontaine and Slade (2007) surveys the empirical literature on vertical integration. Examples of empirical work that examines the competitive effects of vertical integration using reduced form analyses include Waterman and Weiss (1996), Chipty (2001), Hastings (2004), Hastings and Gilbert (2005), Chen and Waterman (2007) and Hortacsu and Syverson (2007). Researchers have also used static structural models to understand the effects of vertical integration (e.g., Brenkers and Verboven (2006); Murry (2015); Asker (2015); Crawford, Lee, Whinston and Yurukoglu (2015)). The model in this paper endogenizes both forward-looking dynamic investment decisions as well as the pricing of intermediate goods. I also contribute to the literature that analyzes innovation with dynamic oligopoly models (Ericson and Pakes (1995), Goettler and Gordon (2011), Borkovsky (2012), Igami (2015) and others) by modeling the com- 
plementarity of innovations between the upstream and downstream firms. The static model of product competition is built on the empirical bilateral bargaining framework developed in Horn and Wolinsky (1988). This type of model has been widely used to analyze the pricing of services and physical goods in vertical industries. Examples include Draganska, Klapper and Villas-Boas (2010), Crawford and Yurukoglu (2012), Grennan (2013), Gowrisankaran, Nevo and Town (2014), Crawford et al. (2015) and Ho and Lee (2016). Like many papers in this literature, I assume that firms in my model use linear price contracts. I later discuss the pros and cons of this modeling choice in more details. Another strand of the empirical structural literature on vertical relations studies the pricing and welfare effects of alternative upstream-downstream relationships (e.g., Villas-Boas (2007); Mortimer (2008); Bonnet and Dubois (2010)).

Compared with the existing literature, the key modeling innovation in this paper is the specification of dynamic upstream and downstream firms in a vertical industry. In addition, I also highlight a data difficulty that Qualcomm's chipset quality is not directly observed. Data only provide quality measures of observed handsets, whereas the relevant measure for Qualcomm's chipset quality is the maximum quality of a phone that a Qualcomm chipset would enable a handset maker to design. I overcome this difficulty by imposing bounds on Qualcomm qualities and using a Simulated Minimum Distance estimator with an inequality constraint. I sample parameters from the estimated confidence set to conduct counterfactual simulations, and the predictions are reasonably precise.

Closely related to this paper, Crawford, Lee, Whinston and Yurukoglu (2015) uses a multistage static model to study how vertical integration affects program carriage choices, prices and ultimately welfare in the US television market. I focus on the dynamic process of innovation, where firms have rational expectations about the future evolution of the industry. In particular, the states and actions of the upstream firm (Qualcomm's quality level and its investment to increase the quality) do not directly affect its own or downstream firms' current period profits, and Qualcomm is solely motivated to innovate by the expectation that downstream firms will innovate and adopt Qualcomm chipsets in the future. Without modeling the forward-looking behavior, explaining why Qualcomm innovates would be hard. In one robustness analysis in Appendix D, I weaken the dynamic incentives by setting the discount rate at 0.5 and obtain the implausible result that increasing the Qualcomm quality level must decrease the innovation cost to rationalize the data.

Road Map The rest of the paper is organized as follows. I first describe the market structure and data in Section 2 . Next, I detail the dynamic model of innovation in Section 3 and the static model of bargaining and pricing in Section 4. Section 5 discusses the estimation of the model. Section 6 reports the counterfactual experiments. Section 7 considers two main robustness checks, and additional robustness analysis is available in Appendix D. 
Table 1: Chipset Origin, \% of Quantity, 2009 to 1st Quarter 2013

\begin{tabular}{cccccc}
\hline & Qualcomm & Samsung & TI & NVIDIA & Other \\
\hline \hline Samsung & 47.55 & 48.96 & 2.63 & 0.61 & 0.25 \\
HTC & 98.30 & 0.00 & 1.48 & 0.08 & 0.14 \\
BlackBerry & 48.15 & 0.00 & 0.00 & 0.00 & 51.85 \\
Motorola & 20.81 & 0.00 & 64.98 & 9.85 & 4.36 \\
LG & 92.67 & 0.00 & 5.37 & 1.96 & 0.00 \\
\hline
\end{tabular}

\section{Industry and Data}

Qualcomm is the most important upstream chipset producer. Qualcomm sells most of its application processor chipsets to non-Apple handset makers, because Apple is vertically integrated and exclusively uses its own chipsets. In 2009, $53 \%$ of non-Apple smartphones sold in the US carried a Qualcomm chipset, and the figure increased to $72 \%$ in the first quarter of 2013 . The price of a chipset is usually between $\$ 16$ and $\$ 40$ (Woyke (2014)). According to reports published by iHS, a tear-down company that tracks component prices, the chipset accounts for $10 \%$ to $20 \%$ of the material cost of a smartphone $\mathrm{S}^{3}$

Qualcomm innovation corresponds with the releases of a new generation of chipsets. A majority of chipsets in the generation Snapdragon S1 were released in October 2008. Qualcomm chipset generations Snapdragon S2, S3 and S4 were released in April 2010, October 2010 and January 2012. Qualcomm Snapdragon S4 is the last generation observed in the data. Products in a later generation feature significant gains in performance (more cores and higher frequency) and energy efficiency.

Qualcomm also competes with several other chipset producers. Qualcomm's main competitors include Samsung's chipset division (South Korea), MediaTek (Taiwan), Texas Instruments (US) and NVIDIA (US). Table 1 reports the origins of chipsets used in major non-Apple handset makers. Compared with other chipset producers, Qualcomm is able to combine more functionalities in its chipsets, and Qualcomm claims that (for example, in Cheng (2012)) this design could enhance performance and extend battery life.

I divide all chipsets into five generations, with the chipsets released before Qualcomm S1 as the 0th generation, and the rest into four generations consistent with Qualcomm chipset generations. I classify non-Qualcomm chipsets based on the number of cores on the chipset, the clock speed of cores and a variety of benchmark scores. Taking January 2009 as month 1, I document when Qualcomm announced the availability of a generation's chipsets and when a handset maker released a phone using that generation's chipset (not necessarily Qualcomm's) in Table 2. Apple relies on its own proprietary chipsets, and a new generation of chipsets is used in a new generation of iPhones. Other handset makers also use the latest chipsets in their new flagship phones, but they rely on Qualcomm at least partially to supply new chipsets for their phones. For example, while Samsung

\footnotetext{
${ }^{3} \mathrm{iHS}$ publishes the material cost estimates of select handsets through its press releases. I have collected some of the published data, which are available upon request.
} 
Table 2: Chipset Announcement and Adoption

\begin{tabular}{lcccc}
\hline & Qualcomm & Apple $^{a}$ & Samsung & HTC \\
\hline \hline Qualcomm S1 or equivalent & -4 & 6 & 6 & 7 \\
Qualcomm S2 or equivalent & 14 & 18 & 19 & 22 \\
Qualcomm S3 or equivalent & 20 & 34 & 33 & 30 \\
Qualcomm S4 or equivalent & 35 & 45 & 43 & 40 \\
\hline
\end{tabular}

Month 1: Jan 2009

${ }^{a}$ : Apple uses its own chipsets and the adoption corresponds with the release of new Apple products.

Figure 1: Product Attribute Trends
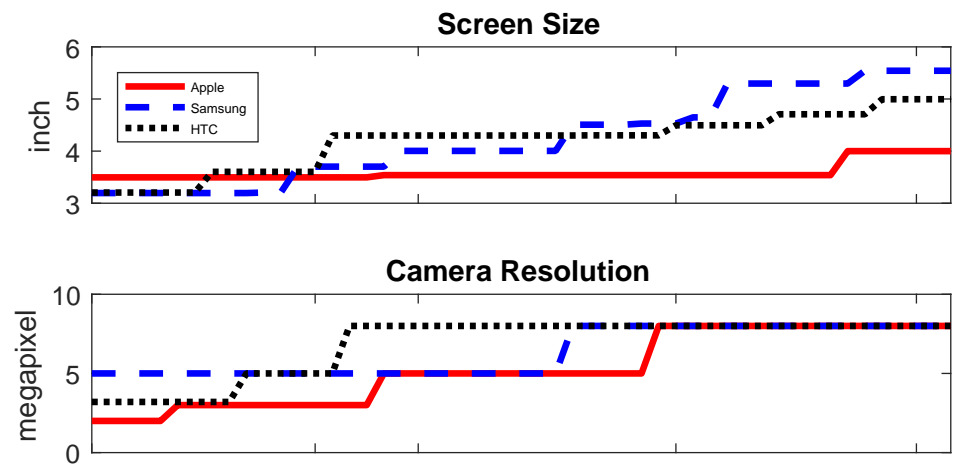

Talk Time

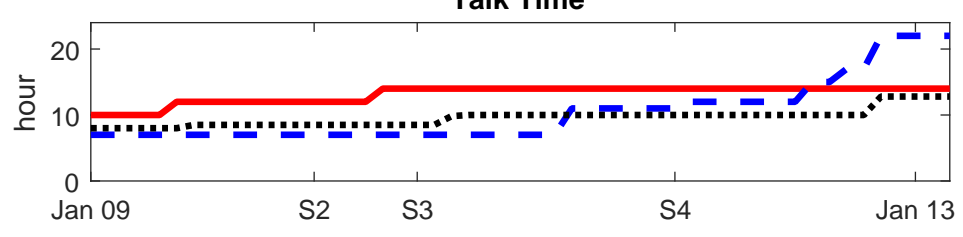

used its own chipsets (Exynos) for Galaxy S2 sold in the US market, Samsung still used Qualcomm chipsets for the same phone sold in China. Although the release timings of these alternative chipsets are not available, Qualcomm usually releases its chipsets more than five months before the release of non-Apple phones that use the same generation's chipsets.

New and better smartphones arrive on the market around the year. Several key dimensions of smartphone qualities, in addition to the generations of chipsets, include the size of the screen (measured by the diagonal length in inches), the resolution of the camera (megapixel) and the maximum talk time (hours) when the phone is fully charged. In Figure 1, I plot the maximum screen size, camera resolution and talk time of all products by Apple, Samsung and HTC in every month. All three measures increase over time.

Smartphone quantity and price data are from ITG Market Research, and the information on a phone's chipset and other characteristics is scraped from technology websites and press releases. 
The data set covers smartphones sold in the US through the four national carriers from January 2009 to March 2013. The observation is at the handset-carrier-month level. In Table 3, I document the retail revenues and quantity sold by the major handset makers for all generations and the more advanced generations. Although BlackBerry sold many low-end handsets in the first year of the data, its sales decreased sharply in later years. Apple, Samsung and HTC account for $70 \%$ of sales (quantity) in the sample, and the top five producers in Table 3 account for $95 \%$. The US market accounts for about 15\% of the global shipment in Q4 2011 (Gartner (2012)), but is likely more important to the high end handset makers. For example, CSIMarket (2014) reports that the US market accounted for 37\% of Apple's revenue in 2014, and this proportion is relatively constant throughout the sample period. In this paper, I assume that the US market accounts for a constant proportion of the world market. Although I do not observe chipset prices directly, I collect the accounting gross margin data of Qualcomm from its quarterly financial reports. The gross margin is defined as

$$
\frac{\text { chipset sales - cost of chipsets }}{\text { chipset sales }}
$$

where the cost of chipsets includes manufacturing, handling, inventory and other costs. Investment and fixed costs (in the accounting sense) are not included. I use the data as the sales-weighted average markup of Qualcomm chipsets. The average markup data allow me to impute productspecific chipset prices, detailed in Section 5. The average gross margin over 17 quarters from January 2009 to March 2013 is $46 \%$, with a maximum of $60 \%$ and a minimum of $33 \%$. Treating the gross margin data as markup allows me to estimate the bargaining model, but there are two potential issues. First, these gross margin data may not reflect the true economic markup. In addition, Qualcomm also sells wi-fi chips and standalone modems, and financial reports do not itemize the gross margins by the types of chipsets. I therefore conduct robustness checks by perturbing the gross margin data in Appendix D. One additional complication is that some of these standalone modems are sold to Apple and other handset makers. I discuss how this issue may affect my analysis in Section 7

Qualcomm owns a large number of patents, and collects royalties as a percentage of a phone's wholesale prices. Because virtually every phone in the world uses Qualcomm's patents, every phone maker pays such fees to Qualcomm, even if the phone maker does not use Qualcomm chipsets. Qualcomm agreed to "Fair, Reasonable and Non-discriminatory" royalty rates when Qualcomm patents were adopted as industry standards and widely used, but differences in fee levels exist (Geradin et al. (2012)). These fees do not enter the gross margin calculation above. I check whether results are robust to the presence of royalties in Section 7 .

\section{A Dynamic Model of Upstream and Downstream Innovation}

Time is discrete $t=1,2, \ldots, T$. The upstream industry consists of Qualcomm and a non-strategic fringe. The downstream industry consists of a finite and fixed set of firms $\mathcal{N}$. I will first discuss the state variables, and their roles in the model will be clear later. The Qualcomm state variable 
Table 3: Total Quantity (Million) and Retail Revenues (\$ Billion)

\begin{tabular}{cccccc}
\hline & \multicolumn{2}{c}{ Quantity } & & \multicolumn{2}{c}{ Retail Revenue } \\
\cline { 2 - 3 } \cline { 5 - 6 } \cline { 5 - 6 } & All Generations & Generation S1-S4 & & All Generations & Generation S1-S4 \\
\hline \hline Apple & 101.35 & 94.79 & & 14.18 & 13.57 \\
Samsung & 38.97 & 37.83 & & 4.60 & 4.51 \\
HTC & 30.58 & 24.67 & & 3.98 & 3.26 \\
BlackBerry & 31.03 & 3.15 & & 3.43 & 0.34 \\
Motorola & 23.28 & 20.55 & & 3.34 & 3.09 \\
LG & 13.68 & 12.83 & & 0.92 & 0.87 \\
\hline
\end{tabular}

Jan 2009 to Mar 2013 on AT\&T, Sprint, T-Mobile and Verizon in US

is the quality frontier $q^{Q}$. The state variables of a handset maker $n$ include the proportion of $n$ 's handsets using Qualcomm and $n$ 's quality frontier, $s^{n}=\left\{\eta^{n}, q^{n}\right\}, \eta^{n} \in(0,1)$. The industry state consists of $s=\left\{t, q^{Q},\left\{\eta^{n}, q^{n}\right\}_{n \in \mathcal{N}}\right\}$. In the empirical game estimated later, $\eta$ 's of Apple and HTC are fixed, and Apple is not constrained by Qualcomm innovation. To simplify the presentation of the model, I assume in this section that all handset makers can adjust their proportions of handsets using Qualcomm, and are constrained by Qualcomm's quality frontier.

In every period, Qualcomm chooses the quality increment of its frontier, $a^{Q} \in\left\{0, \Delta, 2 \Delta \ldots, K_{1} \Delta\right\}$, and the next period Qualcomm state transitions to $q_{t+1}^{Q}=q_{t}^{Q}+a^{Q}$. The action in the data that corresponds with Qualcomm innovation is its release of new chipsets. A handset maker also chooses quality increments $a_{q}^{n} \in\left\{0, \delta, 2 \delta, \ldots, K_{2} \delta\right\}$. If $n$ does not innovate $\left(a_{q}^{n}=0\right)$, the proportion $\eta_{t}^{n}$ stays the same. If $n$ innovates $\left(a_{q}^{n}>0\right), n$ also chooses its proportion of handsets using Qualcomm from a discrete set, $a_{\eta}^{n} \in\left\{\eta_{1}, \eta_{2}, \ldots, \eta_{K_{3}}\right\}$, at the new quality level $q_{t+1}^{n}=q_{t}^{n}+a_{q}^{n}$. n's state transition can be summarized as follows: when $n$ takes action $a^{n}=\left\{a_{q}^{n}, a_{\eta}^{n}\right\}$, the next period state becomes

$$
\begin{cases}q_{t+1}^{n}=q_{t+1}^{n}+a_{q}^{n}, \eta_{t+1}^{n}=a_{\eta}^{n}, & \text { if } a_{q}^{n}>0 \\ q_{t+1}^{n}=q_{t}^{n}, \eta_{t+1}^{n}=\eta_{t}^{n}, & \text { if } a_{q}^{n}=0\end{cases}
$$

The action in the data corresponding with handset maker $n$ 's innovation is the launch of a handset whose quality is higher than any of $n$ 's previous handsets.

The game starts in $t=1$. In every period, firms first receive period profits $\pi_{t}\left(s_{t}\right)$, and make dynamic decisions sequentially. Qualcomm period profit $\pi_{t}^{Q}$ and handset maker period profit $\pi_{t}^{n}$ are given by a pricing game to be detailed in Section 4. Qualcomm moves first, and handset makers move in the sequence $n_{1}, \ldots n_{N}$ :

- Qualcomm draws i.i.d. private cost shock $\varepsilon_{t}^{Q}$, takes action $a_{t}^{Q}$ and pays a sunk cost of $C^{Q}\left(a_{t}^{Q}, \varepsilon_{t}^{Q}\right)$.

- Handset maker $n_{1}$ observes Qualcomm's decision, draws i.i.d. private cost shock $\varepsilon_{t}^{n_{1}}$, takes action $a_{t}^{n_{1}}$ and pays $C^{n_{1}}\left(a_{t}^{n_{1}}, \varepsilon_{t}^{n_{1}}\right)$

$\cdots$ 
- Handset maker $n_{N}$ observes all previous actions, draws i.i.d. private cost shocks $\varepsilon_{t}^{n_{N}}$, takes action $a_{t}^{n_{N}}$ and pays $C^{n_{N}}\left(a_{t}^{n_{N}}, \varepsilon_{t}^{n_{N}}\right)$.

The dynamic optimization problem of Qualcomm in period $t$ solves

$$
\max _{a^{Q}}\left(-C^{Q}\left(a^{Q}, \varepsilon_{t}^{Q}\right)+\beta E\left(V_{t+1}^{Q}\left(s_{t+1}\right) \mid s_{t}\right)\right),
$$

where the expectation is taken over the action probabilities of firms that have not moved in period $t$. The value function of Qualcomm satisfies the Bellman equation

$$
V_{t}^{Q}\left(s_{t}\right)=\pi^{Q}\left(s_{t}\right)+\int_{\varepsilon_{t}^{Q}}\left\{-C^{Q}\left(a^{Q \star}, \varepsilon_{t}^{Q}\right)+\beta E\left(V_{t+1}^{Q}\left(s_{t+1}\right) \mid s_{t}\right)\right\}
$$

where the strategy $a^{Q \star}$ is a function of its own cost shock and the current state. Similarly, a handset maker $n$ solves

$$
\max _{a^{n}}\left(-C^{n}\left(a^{n}, \varepsilon_{t}^{n}\right)+\beta E\left(V_{t+1}^{n}\left(s_{t+1}\right) \mid a_{\mathcal{N}(n)}, a^{n}, s_{t}\right)\right) .
$$

$a_{\mathcal{N}(n)}$ denotes the actions of firms that have moved before $n$. A key component of the model is the constraint that $q_{t}^{n}+a_{q}^{n} \leq q_{t+1}^{Q}$. I use this constraint to capture the complementarity of the upstream and downstream innovations. The Bellman equation of handset maker $n$ is identical to (2), with the superscript $Q$ replaced by $n$. Also note that $n$ 's strategy is a function of its shocks and the actions of firms that have moved. Players in this game have private information and move sequentially, and I solve for the PBE. The last period value function is specified as $V_{T}=\frac{\pi\left(s_{T}\right)}{1-\beta}$.

The innovation cost is specified as

$$
\begin{gathered}
C^{Q}\left(a^{Q}, \varepsilon_{t}^{Q}\right)= \begin{cases}0, & a^{Q}=0 \\
\exp \left(\gamma_{0}^{Q}+\gamma_{1}^{Q} a^{Q}+\sigma^{Q} \varepsilon_{t}^{Q}\right) & a^{Q}>0\end{cases} \\
C^{n}\left(a^{n}, \varepsilon_{t}^{n}\right)= \begin{cases}0, & a_{q}^{n}=0 \\
\exp \left(\gamma_{0}^{n}+\gamma_{1}^{n} a_{q}^{n}-\gamma_{2}^{n} a_{\eta}^{n}+\sigma^{n} \varepsilon_{t}^{q}\right) & a_{q}^{n}>0 .\end{cases}
\end{gathered}
$$

The cost shocks $\varepsilon$ follow the standard normal distribution. I allow the innovation cost of a handset maker to depend on the increment of quality frontier and the choice of proportions of handsets using Qualcomm chipsets.

Although the assumptions of a finite horizon and sequential moves are quite strong, they provide three crucial benefits: (1) the dynamic equilibrium is unique (by backward induction), (2) solving the dynamic game does not involve value function iterations and suffers no convergence problem (Egesdal, Lai and Su (2015)), (3) the finite horizon assumption also helps to capture the nonstationarity in data. The two assumptions have also been used in Igami (2015) for similar purposes. I explore the robustness of both assumptions in Appendix D.

In this model, I assume that dynamic innovation decisions are not contractible. Therefore HTC cannot enter into a contract with Qualcomm about future Qualcomm qualities before Qualcomm's 
innovation is realized. Such contracts would effectively achieve vertical integration. Grossman and Hart (1986), Hart and Moore (1990) and others have shown that without investment coordination, two vertically separated monopolists would invest below the joint profit maximizing level because neither firm fully internalizes the benefit of investment for the other firm. Central to the concept of "incompleteness" in the model above is the difficulty of communicating a firm's innovation decisions to others before the realization of the innovation. While the technology capability of a firm is abstracted into a scalar $q$ in the model, coordinating innovations in the real world potentially would require the chipset maker and handset maker to agree on the joint development of many dimensions of the technology. As discussed in the Introduction, identifying and agreeing to the exact nature of innovation may be hard enough in the face of uncertain future demand and product designs. The legal costs of writing down contracts that enumerate all aspects of cooperative development could be high. Enforcement may be hard, because in the case of contract violations, firms may need to disclose proprietary designs in a legal proceeding. Given these considerations, I assume that firms cannot contract on future innovation.

On the other hand, the ex post enforcement problems may be overcome in an infinite horizon dynamic game, where a PBE may exist such that firms condition strategies on past actions and Qualcomm may be able to credibly delay new chipset releases and "punish" HTC, if HTC does not pay Qualcomm a transfer or commit to Qualcomm chipsets after a Qualcomm innovation. The assumptions of a finite horizon and sequential moves in my model have the effect of a Markov refinement and eliminate the cooperative equilibria. In an infinite horizon game, the folk theorem suggests that upstream and downstream firms can play cooperatively when the discount factor $\beta$ is

sufficiently close to 1 . A body of theoretical literature has examined such cooperative strategies and how the holdup problems manifest differently in a dynamic context (e.g., Halonen (2002); Baker et al. (2002); Che and Sákovics (2004); Che and Sákovics (2007)).

One way to capture coordination in the structural model above is to specify the period profit of HTC as $\varsigma \pi^{Q}+\pi^{H T C}$, where $\varsigma \in(0,1)$ is a reduced form cooperation parameter to be estimated from data. Identifying conduct parameters such as $\varsigma$ requires excluded demand shifters (Bresnahan (1982); Berry and Haile (2014)) or data on innovation costs. In Section 5.2.2. I discuss how investment data can provide information on $\varsigma$.

\section{Bargaining Model}

This section describes a static model of bargaining that determines the profit function $\pi\left(s_{t}\right)$ used as input to the dynamic model. I assume that prices are set in the following order:

1. Qualcomm and handset makers negotiate chipset prices via Nash bargaining.

2. Handset makers take the chipset prices and other components of the marginal cost as given and set wholesale prices.

I start with the demand function. 


\subsection{Consumer Demand}

I model the consumer demand for smartphones using a random coefficient logit model (Berry, Levinsohn and Pakes (1995)). Index consumers by $i$ and handsets by $j$. The utility of consumer $i$ purchasing handset $j$ in period $t$ is

$$
\begin{aligned}
u_{i j t} & =\beta_{0 i} q_{j}-\alpha p_{j t}+\theta_{n(j)}+\kappa_{c(j) t}+\xi_{j t}+\epsilon_{i c j t} \\
& =\underbrace{\bar{\beta}_{0} q_{j}-\alpha p_{j t}+\theta_{n(j)}+\kappa_{c(j) t}+\xi_{j t}}_{\mu_{j t}}+\sigma \nu_{i} q_{j}+\epsilon_{i j t}
\end{aligned}
$$

where $q_{j}=x_{j} \beta$ is the linear quality index of handset characteristics, $\beta_{0 i}$ is a normally distributed scalar random coefficient that captures the heterogeneous tastes for quality: $\beta_{0 i}=\beta_{0}+\sigma \nu_{i}, \nu_{i} \sim$ $\mathcal{N}(0,1), p_{j t}$ is the retail price of the smartphone, $\theta_{n}$ is the handset maker brand fixed effect, $\kappa_{c t}$ is the carrier-year fixed effect plus a quarter fixed effect that captures carrier service heterogeneity and the values of time-varying outside options (this term is referred to as carrier-time fixed effects in the rest of the paper), $\xi_{j t}$ is the unobserved product quality, and $\epsilon_{i j t}$ is an i.i.d. type I extreme value shock. Smartphone characteristics in $x_{j}$ include the screen size, chipset generation fixed effects, camera resolution, weight and battery talk time (the longest time that a single battery charge will last when a user constantly talks on the phone). $\mu_{j t}$ denotes the mean consumer utility, and the utility of no purchase is normalized to zero plus an i.i.d type I extreme value shock $\epsilon_{i \emptyset t}$. The demand for $j$ is given by

$$
D_{j t}=D_{0} \int \frac{\exp \left(\mu_{j t}+\sigma \nu_{i} q_{j}\right)}{1+\sum_{j^{\prime} \in \mathcal{J}_{t}} \exp \left(\mu_{j^{\prime} t}+\sigma \nu_{i} q_{j^{\prime}}\right)} d F_{\nu_{i}}
$$

where $\mathcal{J}_{t}$ is the set of all products available in period $t, D_{0}$ is the market size and $F_{\nu_{i}}$ is the CDF of $\nu_{i}$. I next discuss the pricing of smartphones and chipsets.

\subsection{Smartphones Prices}

Denote the set of handset maker $n$ 's product as $\mathcal{J}_{n t}$. Given the chipset prices $\psi_{j t}$ and other parts of the marginal cost $\omega_{j t}$, handset maker $n$ sets wholesale prices $w_{j t}, \forall j \in \mathcal{J}_{n t}$, to maximize its profit

$$
\sum_{j \in \mathcal{J}_{n t}}\left(w_{j t}-\psi_{j t}-\omega_{j t}\right) D_{j t}
$$

The non-chipset marginal cost of a smartphone is specified as a function of observed characteristics plus a shock:

$$
\omega_{j t} \equiv \underbrace{\lambda_{q} \exp \left(q_{j t}\right)+\lambda_{n(j)}+\lambda_{Q(j)}+\zeta_{c(j) t}}_{\begin{array}{c}
\text { quality, handset maker FE } \\
\text { use Qualcomm? } \\
\text { carrier-time FE }
\end{array}}+\underbrace{\varkappa_{j t}}_{\text {shock }}
$$


To simplify computation, I assume that the carrier subsidy on product $j$ is specified as

$$
r_{j t}=\tilde{\lambda}_{q} \exp \left(q_{j t}\right)+\tilde{\lambda}_{n(j)}+\tilde{\lambda}_{Q(j)}+\tilde{\lambda}_{c(j) t}+\tilde{\varkappa}_{j t}
$$

such that the retail price satisfies $p_{j}=w_{j t}-r_{j t}$. Handset maker $n$ 's profit maximization problem can be re-written as

$$
\max _{p_{j t}, j \in \mathcal{J}_{n t}} \sum_{j \in \mathcal{J}_{n t}}\left(p_{j t}-\psi_{j t}-\left(\omega_{j t}-r_{j t}\right)\right) D_{j t},
$$

and handset makers effectively choose retail prices. To save on notation, I re-define $\omega_{j t}$ as $\omega_{j t}-r_{j t}$, and correspondingly, the coefficients in the non-chipset component $\lambda$ as $\lambda-\tilde{\lambda}$ and the shock $\varkappa$ as $\varkappa-\tilde{\varkappa}$. Equilibrium retail prices satisfy the following first order condition:

$$
D_{j t}+\sum_{j^{\prime} \in \mathcal{J}_{n t}}\left(p_{j^{\prime} t}-\psi_{j^{\prime} t}-\omega_{j^{\prime} t}\right) \frac{\partial D_{j^{\prime} t}}{\partial p_{j t}}=0, \forall j^{\prime} \in \mathcal{J}_{n t} .
$$

In vector notation similar to Eizenberg $(2014)$, the vector of retail prices $p$ satisfies

$$
p-\psi-\omega=(L * \Delta)^{-1} D,
$$

where $L$ is a $\left|\mathcal{J}_{t}\right| \times\left|\mathcal{J}_{t}\right|$ product origin matrix $\left(L_{j j^{\prime}}=1\right.$ if both $j$ and $j^{\prime}$ belong to $\mathcal{J}_{n t}$ and 0 otherwise), $\Delta_{j j^{\prime}}$ is the derivative of the demand for $j^{\prime}$ with respect to the price of $j$, and $*$ represents element-wise multiplication. If the price equilibrium is unique at this stage, the derived demand for chipsets on handset $j$ is well defined. However, there may be multiple Nash-Bertrand equilibria under logit demand with random coefficients and multi-product firms (Echenique and Komunjer (2007)). To select an equilibrium given a set of products $\mathcal{J}_{t}$, I start with the prices of period $t$ 's products whose qualities are closest to those in $\mathcal{J}_{t}$, and apply $(8)$ as a fixed point mapping to solve for the equilibrium prices. In practice, I find that this procedure always converges numerically to a unique price vector $p^{\star}$. Use $\pi_{t}^{n}\left(\boldsymbol{\psi}_{t}\right)$ to denote the downstream firm $n$ 's variable profit in (7) given chipset prices $\psi_{t}$ and $D^{\star}=D\left(p^{\star}\right)$ to denote the derived demand for chipsets.

\subsection{Nash Bargaining and Chipset Prices}

The bargaining game in the first stage of the static game determines the equilibrium chipset prices between Qualcomm and handset makers. I first write down Qualcomm's profit function. Qualcomm earns profits from chipset sales:

$$
\pi_{t}^{Q}(\psi)=\sum_{j \in \mathcal{J}_{Q t}}\left(\psi_{j t}-\underline{\psi}\right) D_{j t}^{\star}
$$

where $\mathcal{J}_{Q t}$ is the set of handsets using Qualcomm chipsets and $\underline{\psi}$ is the marginal cost for Qualcomm to manufacture a chipset.4 Qualcomm negotiates with each handset maker $n$ separately. Denote

\footnotetext{
${ }^{4}$ In reality, Qualcomm does not own any chipset manufacturing facility, and it outsources the production to dedicated fabrication plants.
} 
the vector of chipset prices specific to a Qualcomm- $n$ bargaining pair as $\boldsymbol{\psi}_{n t}=\left(\psi_{j t}, j \in \mathcal{J}_{Q t} \cap \mathcal{J}_{n t}\right)$. The chipset prices are set in a bargaining equilibrium:

Definition. (Nash-bargaining equilibrium) Chipset prices $\boldsymbol{\psi}_{n t}$ for all products in $\mathcal{J}_{Q t} \cap \mathcal{J}_{n t}$ maximize the Nash product corresponding with the bargaining pair of Qualcomm and handset maker $n$, conditional on other chipset prices $\boldsymbol{\psi}_{-n t}$ :

$$
\left[\pi_{t}^{Q}\left(\boldsymbol{\psi}_{n t}, \boldsymbol{\psi}_{-n t}\right)-\tilde{\pi}_{t}^{Q}\left(\boldsymbol{\psi}_{-n t}\right)\right]^{\tau_{t}} \cdot\left[\pi_{t}^{n}\left(\boldsymbol{\psi}_{n t}, \boldsymbol{\psi}_{-n t}\right)-\tilde{\pi}_{t}^{n}\left(\boldsymbol{\psi}_{-n t}\right)\right]^{1-\tau_{t}}
$$

where $\tilde{\pi}$ is the disagreement payoff, and $\tau_{t}$ is the bargaining weight. 5

For $\tilde{\pi}$, I assume that when the negotiation breaks down, the handset maker $n$ uses an alternative functionally identical chipset at a price $\bar{\psi}$ for handsets in $\mathcal{J}_{Q t} \cap \mathcal{J}_{n t}$ and Qualcomm loses revenues from these handsets. Other chipset prices are held fixed and the downstream pricing equilibrium is recalculated. Realistically, the handset quality may also change if a non-Qualcomm chipset is used. I consider this possibility in a robustness check in Appendix D. Based on (9) and the definition of Nash bargaining equilibrium, the vector of all Qualcomm chipset prices $\psi$ satisfies the following first order condition:

$$
\psi=\underline{\psi}+\Theta^{-1} \Phi,
$$

where $\Theta$ and $\Phi$ are defined in Appendix $\mathrm{A}$

The assumption of linear contracts between handset makers and Qualcomm simplifies the contract space and keeps the computation tractable, there are several downsides. First, this assumption introduces double marginalization, an inefficiency that vertical integration can reduce. Based on the Qualcomm gross margin data, I use linear contracts to approximate the variable profits of Qualcomm and handset makers. When the terms in (1) that define the gross margin are close to the true economic primitives and the inefficiency of linear contracts is small compared with the total value of the contract, the approximated variable profits should also be close to the true variable profits. I examine the robustness of the results to potential measurement errors in the gross margin data in Appendix D, and Section 6 demonstrates that the inefficiency of linear contracts is indeed small.

Another concern is whether the linear contract assumption limits firms from achieving innovation coordination. Importantly, even an ex post efficient contract that divides the surplus between the upstream and downstream firms can still lead to under-investment (Grossman and Hart (1986)). As shown in Section 6, the assumption of "ex post negotiation" is the main reason why firms cannot coordinate innovation.

5 Crawford and Yurukoglu (2012) shows that alternative definitions of a bargaining pair do not strongly affect their counterfactual equilibrium price predictions.

${ }^{6}$ There may also be multiple bargaining equilibria. To define the period profit for each firm, I use 10 as a fixed point mapping to iteratively solve for the equilibrium chipset prices, starting from $1.2 \underline{\psi}$. 


\subsection{Period Profit}

Collect the number of products, product qualities, chipset origins and carrier-time fixed effects in a vector $y$. Using the equilibrium selection rules above, Qualcomm and handset maker profits can be written as a function of $y$, demand shocks and marginal cost shocks, $\pi_{t}^{Q}(y, \xi, \varkappa, \tau)$ and $\pi_{t}^{n}(y, \xi, \varkappa, \tau)$. Note that $y$ does not include the state variable of Qualcomm.

In this paper, I focus on how firms adjust quality frontiers, and assume that $y$ is a realization from the distribution $g\left(Y ; \tilde{s}_{t}, \theta\right)$ : the set of products is a random variable that has a stationary distribution conditional on the state variables defined in Section $37^{7}$ where $\tilde{s}_{t}$ is a vector of handset maker quality frontiers and thus a subvector of the full state $s_{t}$ in the dynamic model. The specification of $g(\cdot)$ relies on the empirical distribution of products and described in Appendix B. I further assume that $Y, \xi, \varkappa$ and $\tau_{t}$ are distributed independently. Firms use $\pi^{Q}\left(s_{t}\right) \equiv$ $\pi^{Q}\left(\tilde{s}_{t}\right) \equiv E_{Y, \xi, \varkappa, \tau \mid \tilde{s}_{t}}\left(\pi_{t}^{Q}(Y, \xi, \varkappa, \tau)\right)$ and $\pi^{n}\left(s_{t}\right) \equiv \pi^{n}\left(\tilde{s}_{t}\right) \equiv E_{Y, \xi, \varkappa . \tau \mid \tilde{s}_{t}}\left(\pi_{t}^{n}(Y, \xi, \varkappa, \tau)\right)$ to make dynamic innovation decisions. The assumptions that (1) all firms use the expected profits when making innovation decisions and (2) demand shocks are independent of $Y$ justify the use of BLPtype instruments for demand estimation in the next section.

Using a static model also has another important practical advantage. The assumptions of the static demand and pricing and the stationarity of the product set distribution allow the period profits to be computed separately from the dynamic game. The integration of $\pi_{t}^{Q}(Y, \xi, \varkappa, \tau)$ and $\pi_{t}^{n}(Y, \xi, \varkappa, \tau)$ over the distribution of products, demand shocks, cost shocks and bargaining weights is time-consuming but only needs to be done once, because the random variables are distributed i.i.d. over time. No knowledge of the innovation costs or the dynamic equilibrium is required to compute period profits. The profits are then taken as inputs to the estimation and simulation of the dynamic game. In reality, smartphones are both durable goods and network goods (e.g., Sinkinson (2014); Luo (2016)). Although the framework in this paper does not include dynamic consumers and endogenous network effects, the demand function partially captures both effects with $\kappa_{c t}$, and the model assumes that the two effects are exogenous. The static model also rules out long term contracts. Qualcomm may offer handset makers higher discount to be used in more phones for several periods. The innovation cost parameter $\gamma_{2}^{n}$ in (4) captures this possibility. When $\gamma_{2}^{n}$ is positive, the innovation cost decreases if $n$ uses Qualcomm chipsets on more of its handsets. $\gamma_{2}^{n}$ may reflects Qualcomm's willingness to help a more devoted handset makers to develop products in a more cost-efficient way, but $\gamma_{2}^{n}$ may also represent monetary transfers to handset makers. The limitation is that the transfer is not an endogenous outcome but taken as a structural primitive: $\gamma_{2}^{n}$ may change under an alternative market structure. I do not find counterfactual simulation results sensitive to perturbations to the estimates.

\footnotetext{
${ }^{7}$ See Fan and Yang 2016) for a study on product variety.
} 


\section{Identification and Estimation}

In this section, I discuss the identification and estimation of the bargaining model and the dynamic innovation model. In the bargaining model, there are three sets of structural parameters to be estimated: the consumer preference parameters for smartphones $(\beta, \alpha, \theta, \kappa, \sigma)$ in (5), the smartphone marginal cost parameters $\lambda$ in (6) and the bargaining weight $\tau$ in (9). I calibrate the price of the replacement chipset $\bar{\psi}$ and the marginal cost of manufacturing a chipset $\underline{\psi}$. These parameters together determine the period profit of Qualcomm and handset makers given a set of smartphones and the corresponding demand and marginal cost shocks. The estimated period profit functions are input to the dynamic model, where I estimate the innovation cost parameters $\gamma$ in (3) and (4). Because the estimation of the dynamic model relies on the estimated bargaining model, I discuss the identification and estimation of the bargaining model first.

\subsection{Demand and Smartphone Marginal Cost in the Bargaining Model}

\subsubsection{Identification}

This section discusses the identification of the demand model, the marginal cost function and the bargaining weights. I first explain the identification of the demand. The demand parameters $(\beta, \alpha, \theta, \kappa, \sigma)$ are identified from the joint distribution of the prices, sales and observed smartphone characteristics. The identification may suffer from a sample selection problem because firms choose their product lines. I address this problem with the assumption in Section 4.4. The intuition is that firms do not observe demand and marginal cost shocks until they have made product line decisions 8 Under the assumption that product characteristics are independent of demand shocks, the demand parameters are point-identified and can be estimated with BLP instruments.

I next discuss the identification of the marginal cost parameters $\lambda$ and the bargaining parameter $\tau$. When demand is identified, the pricing equations in (8) identify the markups and hence the smartphone marginal costs as the difference between observed prices and markups. A smartphone's marginal cost is the sum of the chipset price $\psi$ and costs of other components $\omega$. Use $\varrho=\psi+\omega$ to denote this total marginal cost. Both $\psi$ and $\omega$ are not directly observed. Because $\omega$ is a function of phone characteristics and $\lambda$ 's are the coefficients, I need to first invert $\omega$ from $\varrho$. I rely on Qualcomm average chipset markup data and a mapping between $\tau$ and chipset markup. Intuitively, a higher $\tau$ should correspond with a higher average chipset markup in the bargaining equilibrium. The average chipset markup thus identifies $\tau$. Once $\tau$ is known, $\psi$ can be identified as the solution to the bargaining first order condition $(10)$.

I now formalize this intuition. The notation here will also help to illustrate the estimation method guided by the identification strategy. I first restrict the bargaining parameter $\tau$ to be the same for all Qualcomm-handset maker pairs in the months within a quarter (but could be different

across quarters) and $(\underline{\psi}, \bar{\psi})$ are known. This restriction is necessary because I only observe the

${ }^{8}$ A number of papers in the endogenous product characteristics literature (e.g., Eizenberg (2014); Wollmann (2016); Fan and Yang (2016)) use this assumption to facilitate demand estimation. 
average Qualcomm markup aggregated across all handset makers in each quarter 9 Suppose that demand is identified. I next make three uniqueness assumptions that lead to the identification.

First, I assume that the bargaining parameter and non-chipset component costs map uniquely to equilibrium handset prices and chipset prices via the bargaining equilibrium and the Nash-Bertrand equilibrium:

Assumption 1. For every $(\tau, \omega)$, there exists a unique $(p, \psi)$ that satisfies (8) and (10).

Use $\mathcal{H}(\tau, \omega)=(p, \psi)$ to denote this mapping. If the solutions to (8) and $(10)$ for every $\tau$ and $\omega$ are unique, $\mathcal{H}$ is given by this solution. In the case of multiple bargaining or Nash-Bertrand equilibria, the assumption also holds when there is a deterministic equilibrium selection rule known to the researcher, and $(p, \psi)$ is the particular solution to $(8)$ and $(10)$ selected by this rule.10

The next assumption links the observed $(p, \varrho)$ with unobserved $(\tau, \omega)$ :

Assumption 2. Every $(\varrho, p, \tau)$ corresponds with a unique $\psi$ such that

$$
\mathcal{H}(\tau, \varrho-\psi)=(p, \psi)
$$

Such a $\psi$ would be consistent with the observed downstream prices, total smartphone marginal costs and the equilibrium conditions of the bargaining model. This assumption implies that there is a mapping $\tilde{\mathcal{H}}$ such that $\tilde{\mathcal{H}}(\tau, \varrho, p)=\psi$. Therefore fixing $\varrho$ and $p$, a value of the bargaining parameter $\tau$ corresponds with a unique vector of $\psi$ for each market via $\tilde{\mathcal{H}}$. I use $\tilde{\mathcal{H}}$ and data on chipset markup to invert out $\omega$. Specifically, I solve for $\tau$ such that the theoretical average Qualcomm chipset markup in the quarter starting in month $t_{0}$

$$
\frac{\sum_{t=0}^{2}\left(\tilde{\mathcal{H}}\left(\tau, \varrho_{t+t_{0}}, p_{t+t_{0}}\right)-\underline{\psi}\right)^{\prime} \cdot D_{t+t_{0}}}{\sum_{t=0}^{2} \tilde{\mathcal{H}}\left(\tau, \varrho_{t+t_{0}}, p_{t+t_{0}}\right)^{\prime} \cdot D_{t+t_{0}}}
$$

matches the observed quarterly Qualcomm chipset gross margin (described in Section 2).

The last assumption says this $\tau$ is unique:

Assumption 3. (12) is monotonic in $\tau$.

By finding such a $\tau_{t_{0}}$, I also find the corresponding $\psi_{t}=\tilde{\mathcal{H}}\left(\tau_{t_{0}}, \varrho_{t}, p_{t}\right)$ for $t=t_{0}, t_{0}+1, t_{0}+2$. $\varrho_{t}-\psi_{t}$ identifies $\omega_{t}$. Regressing $\omega_{t}$ on the corresponding product characteristics gives $\lambda$.

\subsubsection{Estimation}

I estimate demand using BLP instruments constructed with handset characteristics on the full sample from January 2009 to March 2013. Each month is treated as an independent market.

\footnotetext{
${ }^{9}$ Other papers in the empirical bargaining literature such as Crawford and Yurukoglu (2012) use bargaining-pair specific intermediate prices to estimate pair specific bargaining weights.

${ }^{10}$ The selection rule assumed here is based on the iterative solution to the fixed point problem as in Section 4.2 . Such iterative solution methods do not guarantee the existence of a solution, but I do not encounter non-existence problems in practice.
} 
Table 4: Demand Side Estimates

\begin{tabular}{cccc}
\hline & & Est & Se \\
\hline & Screen Size (inch) & 1 & - \\
& Chipset Generation S1 & 0.460 & 0.113 \\
& Chipset Generation S2 & 0.718 & 0.147 \\
$\beta$ & Chipset Generation S3 & 1.055 & 0.200 \\
& Chipset Generation S4 & 1.674 & 0.280 \\
& Camera Resolution (megapixel) & 0.093 & 0.036 \\
& Weight (gram) & -0.002 & 0.001 \\
$\sigma$ & Battery Talk Time (hours) & 0.056 & 0.013 \\
$\bar{\beta}_{0}$ & Std, Quality & 0.300 & 0.079 \\
$\alpha$ & Mean, Quality & 0.779 & 0.128 \\
$\theta_{n}$ & Price (\$) & 0.007 & 0.002 \\
\hline Carrier year FE, Quarter FE, Samsung, BlackBerry & FE
\end{tabular}

The estimates of the demand model are presented in Table 4 . The characteristics $x_{j}$ used to construct the quality index include the screen size, ${ }^{11}$ chipset generation, camera resolution, weight and the talking time on full battery. The screen size coefficient is normalized to be 1 . The chipset generation fixed effects correspond with Qualcomm's Snapdragon S1 through S4 and comparable products. The omitted generation is for phones that do not use chipsets or use chipsets older than Snapdragon S1. The brand fixed effects of Apple, Samsung and BlackBerry are also included. The demand estimates are reasonably intuitive, with higher generation, camera resolution, lower weights and longer battery talk time contributing positively to the index. A one-hour increase in battery talk time is equivalent to a price decrease of 6.5 dollars for an average consumer. Similarly, a one-megapixel increase in camera resolution is equivalent to a price decrease of 10.9 dollars, while an increase in the screen size by 0.1 inches is equivalent to a price decrease of 11.7 dollars. The estimated standard deviation of consumers' taste for quality is about $40 \%$ of the average taste, suggesting that consumers are heterogeneous in their willingness-to-pay for quality. In our estimation, we include Apple, BlackBerry and Samsung dummies and group all other brands as a baseline brand in the utility function. The Apple brand fixed effect in the demand function is large, worth over $\$ 400$ to consumers. Additional details of the demand estimation are documented in Fan and Yang (2016).

I now discuss how to estimate the marginal cost function (6). Given the estimated demand function and observed prices, the full marginal cost $\omega+\psi$ can be inverted using the first order condition (8). Extra steps are needed to invert out the bargaining parameters $\tau_{t}$ and chipset prices $\psi_{t}$, and estimate $\omega$ as a function of handset characteristics. I fix Qualcomm's marginal cost of manufacturing a chipset to be $\underline{\psi}=\$ 20$, and the cost of non-Qualcomm chipsets at the disagreement point to be $\bar{\psi}=\$ 60$. I base the calibrated value of $\underline{\psi}$ on conversations with fabrication plant

\footnotetext{
${ }^{11}$ The screen size is measured as the diagonal length of the phone, as is standard in this industry, and the unit is inch.
} 
Table 5: Supply Side Estimates

\begin{tabular}{|c|c|c|c|}
\hline & & Est & $\mathrm{Se}$ \\
\hline$\lambda_{q}$ & $\exp ($ quality $/ 10)(\$)$ & 359.251 & 3.641 \\
\hline$\lambda_{Q}$ & Use Qualcomm? (\$) & -21.858 & 0.301 \\
\hline \multicolumn{4}{|c|}{ Carrier year FE, Quarter FE, Apple, Samsung, BlackBerry FE } \\
\hline & & Range & Median \\
\hline$\tau_{t}$ & Bargaining weight & {$[0.28,0.78]$} & 0.47 \\
\hline$\psi_{t}$ & Chipset prices $(\$)$ & {$[28.71,51.29]$} & 35.91 \\
\hline
\end{tabular}

engineers and analysts. $\bar{\psi}$ could be directly estimated if I observe in the data that a handset maker uses different chipsets on the same handset. I do not observe such variations during my sample period. I choose a relatively large $\bar{\psi}$ of $\$ 60^{12}$ to take into account not only the direct cost of buying the alternative chipset, but also potentially the additional cost of equipping a phone with a chipset the phone was not designed to use. I show in robustness checks that further allowing the handset quality to decrease does not change the conclusion very much. The results later will show that even with a large $\bar{\psi}$, which is disadvantageous for handset makers, the potential harm of raising rivals' costs is still limited in the counterfactual vertical integration. To estimate the coefficients in (6), I need to break out the chipset prices $\psi$. To impute $\psi$, I rely on the average Qualcomm markup data in its quarterly financial reports and the mapping $\tilde{\mathcal{H}}$ defined in Section 5.1.1. To calculate $\tilde{\mathcal{H}}$, I solve for a vector of chipset prices $\psi$ consistent with the observed retail prices by iterating (11) for every value of $\left(\tau, \varrho_{t}, p_{t}\right)$. To ensure uniqueness, I always start the iteration from $\psi=1.2 \underline{\psi}$. The gross margin data are quarterly, and I compute a $\tau$ for every quarter by matching 12 with the gross margin in the corresponding quarter ${ }^{13}$ The value of $\tau$ in a quarter enables me to invert out $\psi$ for every month in that quarter. $\psi$ for phones not using Qualcomm is set to 0 . After $\tau$ and $\psi$ are inverted out, I regress $\omega$ on handset qualities, carrier/year FE, quarter FE and brand fixed effects and whether the handset is designed to use Qualcomm chipsets.

Table 5 shows the supply side estimates. The non-chipset components' costs increase with the quality of the smartphone. Using a Qualcomm chipset saves $\$ 22$ in the marginal cost for the nonchipset part of the phone. An alternative interpretation is that if a handset is designed to use a non-Qualcomm chipset, its chipset costs about $\$ 22$. I also present the range of inverted $\tau$ and $\psi$ in Table 5. There are $17 \tau$ 's, and each $\tau$ corresponds with a quarter in the sample. The median Qualcomm chipset price is about $\$ 36$.

There may be several concerns with this approach. One may be concerned that these supply side parameters are not "structural": in a counterfactual vertical integration between Qualcomm and a handset maker, entry into the chipset industry might be expected. First, the foreclosure effect may

\footnotetext{
${ }^{12}$ According to Woyke $(2014)$, most chipset prices are between $\$ 16$ and $\$ 40$.

${ }^{13}$ I use a minimization algorithm to match the model predicted markup with data. I run the algorithm from 10 different starting points and always find a unique solution.
} 
prompt handset makers to seek alternative suppliers. Secondly, because a handset maker may have to reveal proprietary phone designs to Qualcomm during a negotiation, an integrated Qualcomm would have an incentive to exploit this information for its own downstream subsidiary (Allain et al. (2011)). Therefore additional chipset makers may enter to meet the increased demand for Qualcomm alternatives. One may also be concerned with measurement errors in the gross margin data discussed in Section 2. In Appendix D, I consider a robustness test where handset makers face a smaller $\bar{\psi}$ in the counterfactual of vertical integration. I also consider re-estimating the model using perturbed gross margin data.

\subsection{Sunk Cost of Innovation in the Dynamic Model}

The goal is to estimate parameters in (3) and (4). I first use demand estimates to construct handset quality frontiers and the profit functions $\pi^{Q}\left(s_{t}\right), \pi^{n}\left(s_{t}\right)$ defined in Section 4.4 as inputs to the dynamic game. The quality index of a product is constructed as $q_{j}=x_{j} \beta$. I construct the quality frontier of a handset maker in period $t$ as the highest quality of products by $n$ in $t$ : $q_{t}^{n}=\max _{j} q_{j}, j \in \mathcal{J}_{n t}$. By the definition in Section $4.4 \pi^{Q}\left(s_{t}\right) \equiv \pi^{Q}\left(\tilde{s}_{t}\right)$ and $\pi^{n}\left(s_{t}\right) \equiv \pi^{n}\left(\tilde{s}_{t}\right)$ can be simulated with demand estimates.

However, I do not directly observe the quality of Qualcomm chipsets or Qualcomm frontiers. I only observe the latest generation of Qualcomm chipsets according to the announcement dates. The chipset generation fixed effects in the quality index are not the qualities of Qualcomm chipsets. The Qualcomm quality frontier of generation $g$ should be interpreted as the highest quality phone that a handset maker can produce with Qualcomm's generation $g$ chipset. I argue that with appropriate assumptions on the bounds of Qualcomm qualities, one can still make inferences about the underlying cost primitives. First, handset qualities are informative about Qualcomm qualities. Other than vertically integrated Apple, Qualcomm quality is at least as high as the frontiers of other handset makers. Therefore the maximum of non-Apple handset maker frontiers, $\max _{n \neq A p p l e} q_{t}^{n}$ forms the lower bound of the Qualcomm quality frontier in $t$. To bound the Qualcomm quality frontier from above, I make the following assumption:

Assumption 4. When Qualcomm's latest chipset generation is $g$ in period $t$, Qualcomm quality $q_{t}^{Q}$ is less than the quality of the first non-Apple handset using generation $g+1$ Qualcomm chipset.

For example, Qualcomm's latest generation is S3 in November 2011, and in my data set, the first phone that uses the next generation S4 chipsets is One S by HTC (available in March 2012) with quality index 6.88. Therefore under the assumption above, Qualcomm quality in November 2011 is less than 6.88, and no non-Apple handset maker can produce a phone with quality higher than 6.88 until S4 becomes available. In the data, Qualcomm added new varieties of chipsets to the Snapdragon S3 product line over time. Some of the new additions enabled some handset makers to produce phones whose qualities are higher than 6.88, but Qualcomm only added these chipsets after the launch of Snapdragon S4 and the assumption above is still valid. 


\subsubsection{Identification}

I use the estimated period profit function, handset maker quality frontiers $q_{t}^{n}$ over time, proportions of a handset maker's models using Qualcomm chipsets $\eta_{t}^{n}$ over time and Qualcomm's upper bounds given by Assumption 4 to identify the innovation costs. Because Qualcomm's quality is observed up to a range, the innovation cost parameters are only set identified. I argue that the observed quality improvements can still provide meaningful bounds on structural parameters. For handset maker $n$, if $\gamma_{0}^{n}$ is too large compared with the expected increase of the discounted sum of $n$ 's period profits when $n$ 's quality frontier is increased, $n$ would innovate infrequently. Similarly, a large $\gamma_{1}^{n}$ reduces the size of each innovation. On the other hand, a large $\gamma_{2}^{n}$ allows $n$ to innovate faster when it uses more Qualcomm chipsets. Assumption 4 bounds $\gamma^{Q}$ from below: if $\gamma^{Q}$ is too small, Qualcomm's quality would increase too much and violate the upper bounds of Qualcomm's quality. The observed non-Apple handset maker quality frontiers bound $\gamma^{Q}$ from above: a large $\gamma^{Q}$ reduces Qualcomm's innovation, and because non-Apple handset makers cannot innovate above Qualcomm's frontier, the handset maker quality frontiers would be lower than the observed if $\gamma^{Q}$ is too large. The bounds also restrict the variance of innovation costs. If $\sigma^{Q}$ is large, Qualcomm would take large innovation jumps with high probability, because its innovation cost would be low whenever $\varepsilon^{Q}$ is negative. Qualcomm's innovation rate then would be higher than the bounds in Assumption 4. The bounds also restrict $\sigma^{n}$. A large handset maker $\sigma^{n}$ would similarly increase the innovation rate of a handset maker. Because handset makers at higher quality levels generate more profits for Qualcomm and a high Qualcomm quality frontier enables handset makers to innovate, a large $\sigma^{n}$ increases the future value of a Qualcomm innovation. Therefore a very large $\sigma^{n}$ could also cause Qualcomm's frontier to be too high and violate the constraint. The data on handset maker quality improvements and the upper bounds of the Qualcomm quality levels thus provide two-sided bounds on all structural parameters.

\subsubsection{Estimation}

I use a Simulated Minimum Distance estimator with one inequality constraint to recover a confidence set for the innovation cost parameters. For any vector of innovation cost parameters, I am able to solve for the unique Perfect Bayesian Equilibrium at a discount rate of 0.99. To limit the computational burden, I estimate a dynamic game of Qualcomm and the top three handset makers from 2010 to 2013: Apple, Samsung and HTC. In Section 7 I include BlackBerry, Motorola and LG but assume that their quality frontiers are exogenous conditional on HTC and Samsung's quality frontiers. When solving the dynamic game, I assume that the order of moves is Qualcomm, Apple, Samsung and HTC. Appendix D considers the case where the order of handset maker moves is

reversed. Consistent with data, Apple is assumed to always use non-Qualcomm chipsets $\left(\eta^{A}=0\right)$ and is not constrained by the Qualcomm quality frontier; HTC innovation is constrained by Qualcomm, and always chooses $\eta^{H T C}=1$ : the chipsets of all HTC phones are supplied by Qualcomm and their prices are determined in the bargaining equilibrium; Samsung innovation is also constrained by Qualcomm, but can adjust the proportion of Qualcomm chipsets used on Samsung 
handsets. To guard against the effect of the finite horizon assumption, the model is solved by backward induction from six months after the last period of the data, September 2013. In Appendix D, I further check the sensitivity of the finite horizon assumptions by solving the game from March 2014. The carrier-time fixed effects of April 2013 to March 2014 are extrapolated from demand estimates in earlier periods. To accommodate the potential heterogeneity in the sunk cost functions (3) and (4), I estimate a firm specific $\gamma_{0}$ and $\gamma_{1}$. I restrict $\sigma^{\text {handset }} \equiv \sigma^{\text {Apple }}=\sigma^{\text {Samsung }}=\sigma^{\text {HTC }}$ and estimate a different $\sigma^{\text {Qualcomm }}$, giving me a total of 11 parameters to estimate. There are a total of 51 months of data. I fix the qualities in month 1 and use quality choices of the next $T=50$ periods for estimation. I use a computationally simple estimator in Shi and Shum $(2015)$ to find the $95 \%$ confidence set of the identified set. Denote the equality moments as $g^{e}$ and inequality moments as $g^{i e} \leq 0$. The confidence set is defined as

$$
C S_{T}=\left\{\theta \in \Theta: g^{i e} \leq 0, g^{e} / W g^{e} \leq \chi_{d}^{2}(0.95) / T\right\}
$$

where $W$ is the weighting matrix and $\chi_{d}^{2}(0.95)$ is the $95 \%$ quantile of $\chi^{2}$ distribution of $d$ degrees of freedom. $d$ is the number of equality constraints in $g^{e}$. Like Goettler and Gordon (2011), I match the following stationary equality moments in simulation and data:

1. mean innovation rates, defined as $\left(q_{51}-q_{1}\right) / T$ for Apple, Samsung and HTC;

2. mean proportion of Qualcomm chipsets on Samsung products, $\sum_{t=2}^{51} \eta_{t} / T$.

Denote the upper bound of Qualcomm quality observed in each period $t$ as $\rho_{t}$. Use $q_{t, r}^{(\cdot)}$ and $R$ to denote the quality in simulation $r$ and the total number of simulations. Assumption 4 implies $q_{t}^{Q}<$ $\rho_{t}$. To create a stationary inequality moment, I subtract the the highest non-Apple smartphone quality from Qualcomm quality and consider the restriction

$$
q_{t}^{Q}-\max \left(q_{t}^{S a m}, q_{t}^{H T C}\right)<\rho_{t}-\max \left(q_{t}^{S a m}, q_{t}^{H T C}\right) .
$$

The actual inequality constraint used in estimation is

$$
\sum_{r=1}^{R} \sum_{t=2}^{51}\left(q_{t, r}^{Q}-\max \left(q_{t, r}^{S a m}, q_{t, r}^{H T C}\right)\right) / R T \leq \sum_{t=2}^{51}\left(\rho_{t}-\max \left(q_{t}^{S a m}, q_{t}^{H T C}\right)\right) / T .
$$

I detail the model solution, estimation and simulation procedure in Appendix C.

There are 5 moments for 11 parameters. I leave the model under-identified for several reasons. First, these moments are closely related to the identification of the mean innovation costs. For example, high $\gamma_{0}$ or $\gamma_{1}$ for the handset makers will imply slow innovation and cause deviations in the first set of equality moments. High Qualcomm $\gamma_{0}$ or $\gamma_{1}$ will also imply slow Qualcomm innovation, which also slows down Samsung and HTC innovation. If Qualcomm $\gamma_{0}$ or $\gamma_{1}$ are low, Qualcomm innovates more quickly and will violate the inequality constraint. The remaining Qualcomm usage parameter $\gamma_{2}^{n}$ is identified by the second equality moment. Secondly, the functional form restriction and the dynamic equilibrium strategies imply a tight relationship between the four firms' innovation 
Table 6: Estimates of Innovation Costs

\begin{tabular}{|c|c|c|}
\hline \multicolumn{3}{|c|}{ 95\% Confidence Set } \\
\hline \multirow{4}{*}{$\gamma_{0}$} & Apple & {$[0.36,0.84]$} \\
\hline & Samsung & {$[-0.83,1.05]$} \\
\hline & HTC & {$[-0.36,0.61]$} \\
\hline & Qualcomm & {$[-4.86,-4.64]$} \\
\hline \multirow{4}{*}{$\gamma_{1}$} & Apple & {$[16.02,17.52]$} \\
\hline & Samsung & {$[8.08,13.18]$} \\
\hline & HTC & {$[8.38,10.40]$} \\
\hline & Qualcomm & {$[6.03,7.17]$} \\
\hline$\gamma_{2}$ & Samsung & {$[4.10,5.12]$} \\
\hline \multirow{2}{*}{$\sigma$} & Handset & {$[4.13,5.08]$} \\
\hline & Qualcomm & {$[0.34,0.61]$} \\
\hline \multicolumn{3}{|c|}{$\begin{array}{l}\text { I report the min and max of each parameter in } \\
\text { the confidence set. The confidence set consists } \\
\text { of a set of vectors of parameters that satisfy }\end{array}$} \\
\hline
\end{tabular}

rates as well as a tight relationship between the innovation rates and other features of the innovation paths, such as the variance of the innovation rates. Adding additional moments rejects the current model, while a more flexible functional form of the innovation cost function would add to the high computational cost of a simulation-based estimator. Moment inequality methods that allow for model mis-specification (Chernozhukov et al. (2007)) and other alternatives (e.g., Andrews and Soares (2010); Romano et al. (2014)) involve a bootstrap step to compute the confidence set and are not computationally feasible. Balancing the computational feasibility and model flexibility, I choose the former, focusing on matching the moments most important to the research question and taking advantage of the computationally simple set estimator in Shi and Shum (2015).

The estimated $95 \%$ confidence set consists of a set of vectors of parameters that satisfy 13 . I report the minimum and maximum of each parameter in the confidence set in Table6. Because the cost functions are specified as an exponential function of a linear combination of innovation actions, we can interpret the parameter estimates as a "semi-elasticity". For example, increasing quality by 0.1 unit increases the innovation cost by 1.6 to 1.7 times for Apple. Using Qualcomm chipsets for all handsets reduces the innovation sunk costs for Samsung. The magnitude of the handset maker private shock is large. In Figure 2, I plot the brand-fixed effect adjusted quality frontiers $\left(q_{t}^{n}+\frac{\theta_{n}}{\bar{\beta}_{0}}\right)$ in data and simulation. The simulated quality frontier is the average of 960 simulated paths based on a random draw of parameters in the confidence set.

I use simulation to interpret the estimates in terms of the levels of innovation costs. I use a procedure described in Appendix $\mathrm{C}$ to sample a representative set of points from the confidence set to simulate the dynamic model. The model is simulated at these parameter values for 960 times for the sample period. Table 7 reports the range of the simulated total investment expenditures of 
Data

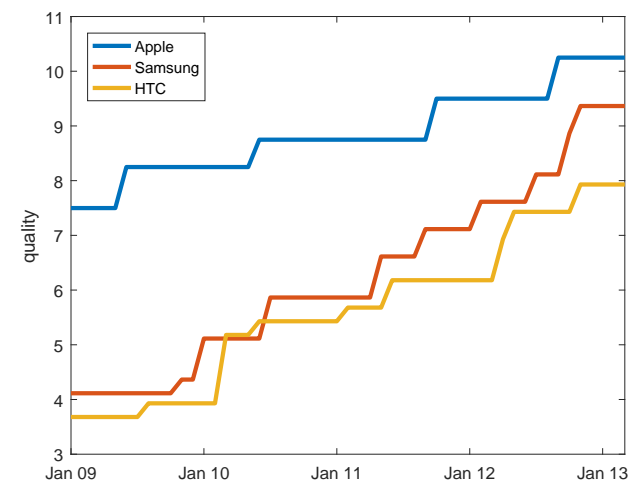

Figure 2: Quality Frontier Evolution

Simulation

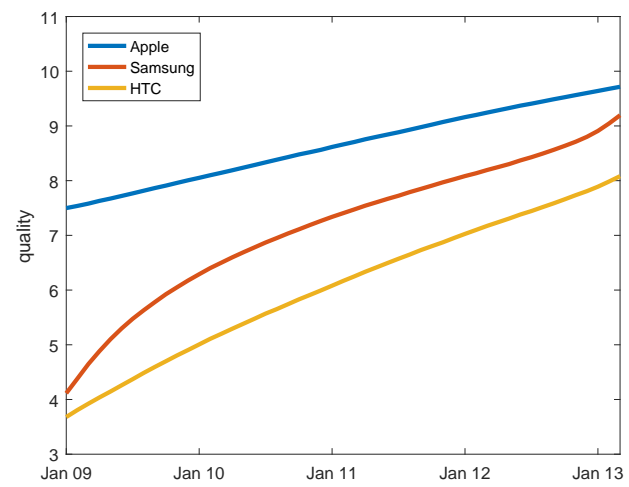

I plot the brand-fixed effect adjusted quality frontiers $q_{t}^{n}+\frac{\theta_{n}}{\overline{\beta_{0}}}$ in data and simulation. The simulated quality frontier is the average of 960 simulated paths based on a random draw of parameters in the confidence set. The vertical axis is in the unit of quality indices constructed from the demand estimates.

Apple, Samsung, HTC and Qualcomm across sampled points in the confidence set. The reported range approximates the $95 \%$ confidence interval of the model prediction. To examine whether these figures are sensible, I sum up the operating expenses (R\&D, selling, general and administrative costs but not manufacturing costs of the goods sold, in the accounting sense) in HTC's financial reports, discounted by an annual rate of $0.99^{12}=0.89$. Apple, Samsung and Qualcomm have major operations outside the application chipset and smartphone industries, and their accounting costs are less relevant. The discounted HTC operating expenses are 6.83 billion dollars during the period. According to HTC's annual reports, $51 \%$ of HTC revenues come from North America. Under the assumptions that the US market accounts for a constant share of the world market and the US market accounts for the majority of HTC's North American revenues, the total simulated HTC investment scaled to the global level will be at least as high as the range of 6.14 to 9.50 billion dollars. The simulated investment level matches HTC accounting figures in scale.

One may also view the comparison as evidence for a model of incomplete contracts. As discussed in Section 3, to allow for cooperative strategies, I can specify HTC period profit as $\varsigma \pi^{Q}+\pi^{H T C}$ and estimate $\varsigma$. Investment data can be informative about $\varsigma$. If cooperation increases innovation and hence total investment, then innovation rates and investment levels are higher when $\varsigma=1$ than when $\varsigma=0$. If the quality choice data are generated by a model of $\varsigma=1$, my estimates under the assumption that $\varsigma=0$ would incorrectly attribute the high levels of innovation to low innovation costs instead of cooperation, and the simulated HTC innovation costs would be lower than the actual investment. In the above, the range of simulated investment levels (6.14 to 9.6 billion dollars) is not below the observed investment level of 6.83 billion dollars. If higher quality firm specific investment data were available, I could formally estimate $\varsigma$. 
Table 7: Simulated Investment Range (\$ Billion), Jan 2009 to March 2013

\begin{tabular}{cc}
\hline & Investment \\
\hline \hline Apple & {$[6.72,7.74]$} \\
Samsung & {$[1.84,3.31]$} \\
HTC & {$[3.07,4.75]$} \\
Qualcomm & {$[0.61,0.86]$} \\
\hline
\end{tabular}

\section{Counterfactual Simulation}

I investigate the effects of a Qualcomm-HTC merger. HTC is a natural choice for this counterfactual because of its high dependence on Qualcomm chipsets. Moreover, Apple, the unconstrained handset maker, and Samsung, which can flexibly adjust the proportion of its handsets using Qualcomm, resemble typical downstream competitors to a vertically integrated firm. Samsung may decrease the use of Qualcomm chipsets because of the raising rivals' cost effect, but could also increase the use of Qualcomm chipsets to reduce innovation costs. I simulate the effects of vertical integration and decompose the effects into the investment effects and price effects. I simulate every counterfactual scenario 240 times for the period of January 2009 to December 2011 at points sampled from the confidence set as described in Appendix C. I only simulate the first 36 periods because firms are less forward looking as time moves closer to the last period of the theoretical model. All dollar figures are discounted to January 2009.

Vertical integration has two effects. First, the integrated firms invest to maximize the joint value function, internalizing the marginal effect of HTC innovation on Qualcomm and vice versa. Secondly, the integrated firms also jointly set prices, reducing double marginalization but potentially raising rivals' costs. In all simulations, firms still move sequentially: the "Qualcomm division" of the merged firm still is the first firm to observe its private shock and move, followed by Apple, Samsung and the "HTC division" of the merged firm. I do so to keep the information structure intact so that the simulations under various market structures are comparable. Alternatively, one could allow HTC to observe its shock and move earlier because of the merger. However, the simulated counterfactual effect under this scenario would contain both the effect of the market structure change as well as the change in the sequence of the move. Because the sequential move assumption is purely a device to simplify the model, I keep the same move sequence in estimation and counterfactual simulation to focus on the effect of the market structure change. The new dynamic programming problems for Qualcomm and HTC divisions of the merged firm thus become

$$
\begin{gathered}
\max _{a^{Q}}\left\{-C^{Q}\left(a^{Q}, \varepsilon^{Q}\right)+\beta E\left(V_{t+1}^{V I}\left(s_{t+1}\right) \mid a^{Q}, s_{t}\right)\right\} \\
\max _{a^{H T C}}\left\{-C^{H T C}\left(a^{H T C}, \varepsilon^{H T C}\right)+\beta E\left(V_{t+1}^{V I}\left(s_{t+1}\right) \mid a^{Q}, a_{\mathcal{N}(H T C)}, s_{t}\right)\right\},
\end{gathered}
$$

and the Bellman equation for the joint firm is

$$
V_{t}^{V I}\left(s_{t}\right)=\tilde{\pi}^{V I}+E\left(-C^{Q}\left(a^{Q \star}, \varepsilon^{Q}\right)-C^{H T C}\left(a^{H T C \star}, \varepsilon^{H T C}\right)+\beta V_{t+1}^{V I}\left(s_{t+1}\right) \mid s_{t}\right),
$$


where the expectation is taken over $\left(\varepsilon^{Q}, \varepsilon^{H T C}\right)$, the corresponding strategies of Qualcomm and HTC, and the action probabilities of their rivals. $\tilde{\pi}^{V I}$ is the sum of $\tilde{\pi}^{Q}$ and $\tilde{\pi}^{H T C}$, the joint equilibrium profit under vertical integration. The first order conditions that define the new equilibrium prices in the static pricing game are outlined in Appendix A

I conduct three sets of simulations: no vertical integration, investment coordination only and full VI with both investment and price effects. The purpose of the second simulation is to parse out the investment effect. Specifically, I simulate the outcomes where Qualcomm and HTC price their products as if they were still separate, but the two firms pool their profits when making dynamic investment decisions: i.e. the investment decisions of Qualcomm and HTC are solutions to 15 , but I replace $\tilde{\pi}^{V I}=\tilde{\pi}^{Q}+\tilde{\pi}^{H T C}$ with $\pi^{Q}+\pi^{H T C}$ in 16 . The difference between this simulation and the "no VI" simulation shows the net investment effects, whereas the difference between the "investment coordination" and the "full VI" simulations shows the additional price effects. The equivalent of "investment coordination" in reality is a research consortium, where product development is jointly conducted between firms but pricing is independent.

Table 8 reports the simulation results. The end points of the intervals are the maximum and minimum of the simulation outcomes across points sampled from the confidence set according to the procedure in Appendix C. The column "No VI" reports the range of simulation results at the observed market structure, and the column "Investment Coordination" reports the results where Qualcomm and HTC coordinate innovation but not pricing. The column "Full VI" reports the results where Qualcomm and HTC coordinate both investment and pricing. The numbers under "Difference \%" represent the changes in outcomes under different market structures but at the same vector of parameters. I calculate the percentage changes for all parameters in the sampled set of parameters and report the minimum and maximum.

I first summarize the main findings:

1. Vertical integration increases the innovation of both upstream and downstream firms; the changes in Apple's innovation rates are comparatively much smaller.

2. Consumer surplus and total surplus increase.

3. The investment effects on the innovation rates of Qualcomm, HTC and Samsung and the surpluses are larger than the price effects.

4. Price effects decrease HTC's retail prices and increase the prices of Qualcomm chipsets sold to Samsung.

Next, I discuss the intuition behind these observations in Table 8. The increase in Qualcomm and HTC innovation is intuitive, because they internalize the marginal value of innovation on each other. To visualize the magnitude of the effect, I examine the first order difference of value functions, $V^{n}\left(q^{n}+0.25, q^{n^{\prime}}\right)-V^{n}\left(q^{n}, q^{n^{\prime}}\right)$, where $n, n^{\prime} \in\{$ Qualcomm, HTC $\}$. The difference represents the dynamic marginal value of innovation. I plot these differences in Fig. 3 for the month of January 2011, where Apple and Samsung quality levels (brand fixed effect adjusted) are fixed at 8.2 (iPhone 
Figure 3: Marginal Effects of Qualities on HTC and Qualcomm Value Functions

Increase in HTC Value Function When HTC

Quality Increases by 0.25

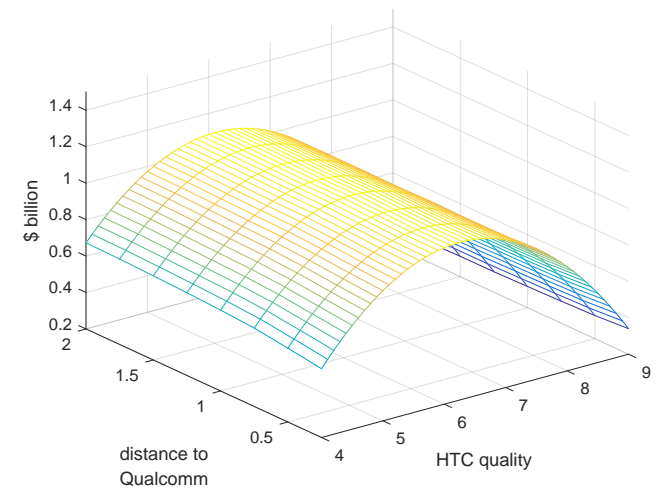

Increase in Qualcomm Value Function When HTC Quality Increases by 0.25

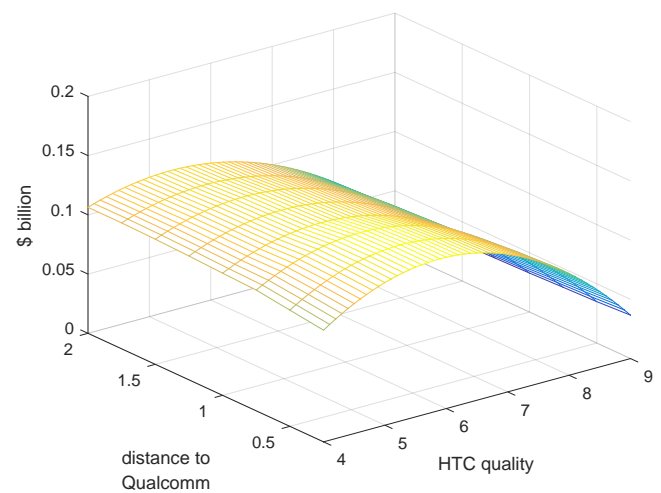

Increase in HTC Value Function When Qualcomm Quality Increases by 0.25

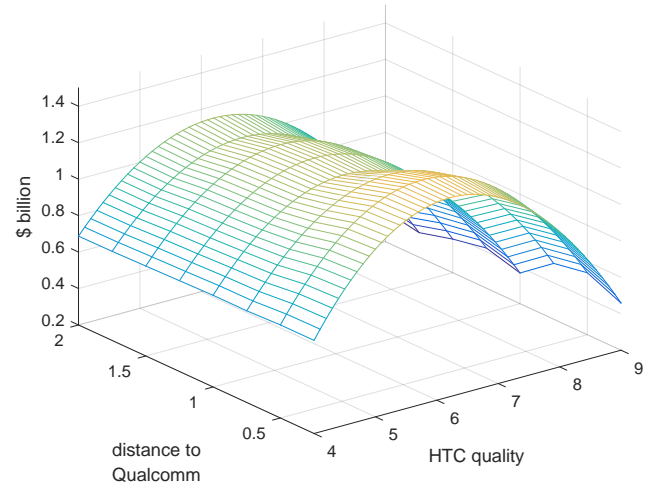

Increase in Qualcomm Value Function When Qualcomm Quality Increases by 0.25

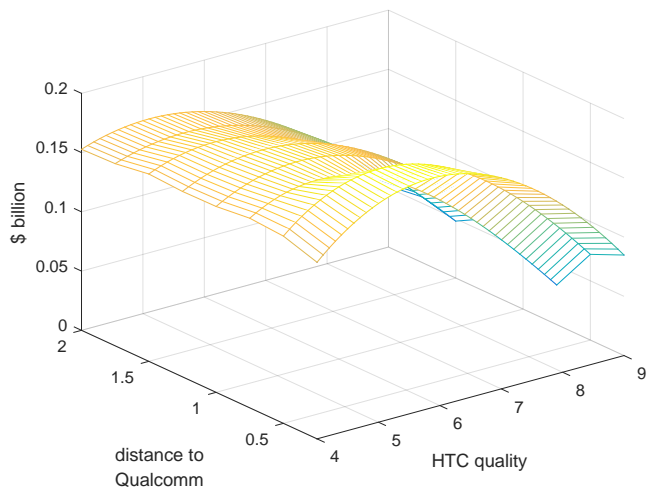

The axis "distance to Qualcomm" is defined as $q^{\text {Qualcomm }}-q^{\text {Samsung }}$.

3GS) and 6.7 (Galaxy S II). The axis named "distance to Qualcomm" denotes $q^{\text {Qualcomm }}-q^{H T C}$. The value functions are computed based on a randomly selected point in the $95 \%$ confidence set. Per unit increase of Qualcomm or HTC quality, HTC value function would increase in the range of 0.4 to 1.2 billion dollars. Per unit increase of HTC quality increases Qualcomm value function by 0.05 to over 0.15 billion dollars, and the effect of Qualcomm's own quality change is slightly larger.

Samsung also innovates faster and is less constrained by Qualcomm. Although the average number of months that $q^{\text {Samsung }}=q^{Q}$ is only reduced from 4.78 months to 4.50 months, I argue that the harm of being constrained by Qualcomm is significantly lessened. I illustrate this point by examining the second order difference of Samsung's value function. Suppose that Samsung is constrained and cannot innovate, but would otherwise find it profitable to innovate if it were not constrained, then by allowing Samsung to innovate, Qualcomm innovation should have a large and 
Figure 4: Marginal Effect of Qualcomm Quality on the Marginal Value of Samsung Innovation

No VI

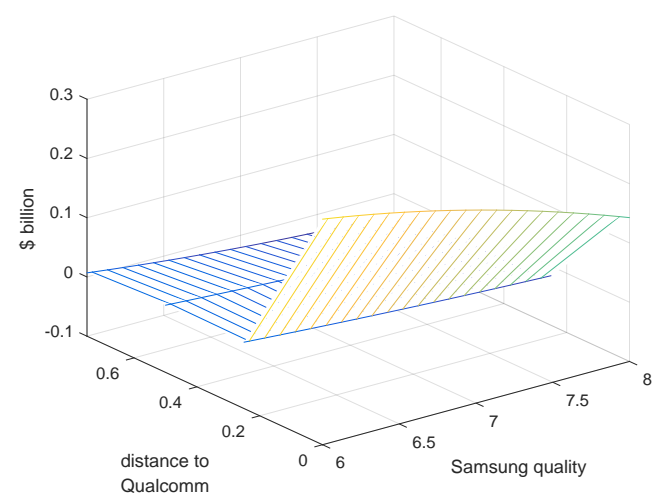

Qualcomm-HTC VI

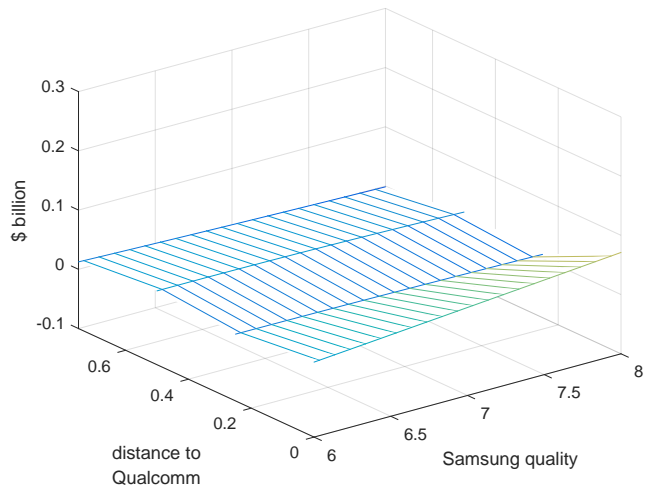

The axis "distance to Qualcomm" is defined as $q^{\text {Qualcomm }}-q^{\text {Samsung }}$.

positive impact on the marginal value of Samsung innovation, which means that

$$
\begin{array}{r}
V^{\text {Samsung }}\left(q^{\text {Samsung }}+0.25, q^{\text {Qualcomm }}+0.25\right)-V^{\text {Samsung }}\left(q^{\text {Samsung }}, q^{\text {Qualcomm }}+0.25\right) \\
-\left(V^{\text {Samsung }}\left(q^{\text {Samsung }}+0.25, q^{\text {Qualcomm }}\right)-V^{\text {Samsung }}\left(q^{\text {Samsung }}, q^{\text {Qualcomm }}\right)\right)
\end{array}
$$

should be large and positive when $q^{\text {Samsung }}=q^{\text {Qualcomm }}$. A larger value implies greater harm from the constraint, because Samsung has to delay a profitable innovation. In Fig. 4. I plot this second order difference for Samsung when Apple and HTC qualities (brand fixed effect adjusted) are fixed at 9 and 6 . In the "No VI" scenario, the second order derivative is indeed positive and large when the distance to Qualcomm frontier $\left(q^{\text {Qualcomm }}-q^{\text {Samsung }}\right)$ increases from 0, suggesting that Samsung is likely to miss profitable innovation opportunities when constrained. The value of the innovation that Samsung fails to capture because of the constraint is economically large: the spike in the left figure accounts for $20 \%$ to $40 \%$ of the corresponding marginal value of Samsung innovation. In the VI counterfactual, the magnitude of the second order difference is smaller. The results show that the overall investment effect of vertical integration on Samsung, which includes the effect from the lessened constraint and the competitive effect, is positive. Samsung also uses more Qualcomm chipsets, reducing the innovation costs.

Furthermore, vertical integration increases consumer and producer surplus. Most of the increase come from the faster innovation, but the decrease in HTC prices also helps to increase consumer surplus. In particular, while higher quality products may be priced higher, eliminating double marginalization reduces HTC retail prices by about $6 \%$ even when price effects increase HTC innovation. Qualcomm also increases the prices of the chipsets sold to Samsung, but the overall welfare effect is positive. 


\section{Robustness Analysis}

I consider two main robustness checks in this section. The first check considers Qualcomm's royalty income. The second check considers the effect of additional handset makers $\tilde{\mathcal{N}}=\{$ BlackBerry, Motorola, LG\}. More robustness checks are available in Appendix D.

In the first robustness check, I allow Qualcomm to collect patent royalties from handset makers. To model the effect of royalty fees, I first construct a measure of wholesale prices. I collect additional data on the average service prices consumers pay to carriers. Consumers during the sample period are typically on two-year contracts, and I use the retail phone price $p_{j}$ plus the discounted sum of consumer service payments $v_{t}$ as the total carrier revenue per customer. The average of $v_{t}$ in my calculation is 855 dollars. A wireless carrier whose main businesses are wireless and data services (like T-Mobile) typically has a gross margin of $50 \%$ according to their financial reports. Assuming that the wholesale prices of the phones are the only marginal costs carriers face, I measure the

wholesale price as $w_{j t}=\frac{1}{2}\left(p_{j t}+v_{t}\right)$. Given the royalty rate $z_{n}$ for handset maker $n$, the handset maker profit function becomes

$$
\max _{p_{j t}, j \in \mathcal{J}_{n t}} \sum_{j \in \mathcal{J}_{n t}}\left(\left(1-z_{n}\right) \cdot \frac{1}{2}\left(p_{j}+v_{t}\right)-\psi_{j t}-\omega_{j t}\right) D_{j t},
$$

and the Qualcomm profit function is modified to include the additional revenues from royalties. At the disagreement point in the bargaining game, Qualcomm continues to earn royalties from the handset maker even if the handset maker switches to an alternative chipset. I assume that Apple's royalty rate is 2\%, Samsung 3\% and HTC 5\% (Arghire (2009); Clark (2009)).

In the second robustness check, I add in products by firms in $\tilde{\mathcal{N}}$. To maintain computational tractability, I assume that quality frontiers of firms in $\tilde{\mathcal{N}}$ are exogenous conditional on the maximum of HTC and Samsung frontiers. Given $\tilde{s}_{t}=\left(q_{t}^{\text {Apple }}, q_{t}^{\text {Samsung }}, q_{t}^{H T C}\right)$, I compute a firm's profit function as

$$
E\left(\pi_{t}\left(\tilde{s}_{t},\left(q_{t}^{k}\left(\tilde{s}_{t}\right)\right)_{k \in \tilde{\mathcal{N}}}\right)\right)
$$

where the frontier of $k \in \tilde{\mathcal{N}}$ is a function of $\tilde{s}_{t}$, and the associated set of products is a random variable to be integrated out. The procedure is described in Appendix B. One complication is that the number of products by firms in $\tilde{\mathcal{N}}$ changes drastically over time in data. LG has one product at the start of the sample and over 10 at the end, whereas BlackBerry's product line exhibits the opposite trend. Because the goal of this exercise is to check whether the dynamic implication of a vertical merger is sensitive to additional static competition in the product space, the sampling procedure in Appendix B ignores this non-stationarity and on average includes more and higher quality products from $\tilde{\mathcal{N}}$ than observed in data.

The estimates of the two robustness checks are reported in Table 9. The simulation results are reported in Tables 10 and 11. The estimated $\gamma_{1}$ for Qualcomm in the first robustness check is much larger than in Table 8 . The estimates of the second robustness check are similar to the main specification. In the counterfactual simulations, the qualitative patterns of both specifications are 
broadly consistent with the main specification. The counterfactual innovation rates increase less in the second robustness check. The producer surplus for this robustness check includes the variable profits of firms in $\tilde{\mathcal{N}}$.

Qualcomm chipsets are bundled with modems, but Qualcomm also sells standalone modems to handset makers, including Apple. Qualcomm thus internalizes the value of Apple innovations more than what the model captures. However, it is important to recognize that the modem sales affect Qualcomm innovations in a way similar to Qualcomm royalty revenues, and in some instances, Qualcomm only collects royalties from some clients and sells them modems for free (Mock (2005)). Because no detailed data are available on the modem manufacturers for phones in my sample, I did not conduct analysis regarding modem sales.

\section{Discussion and Conclusion}

This paper estimates a new model that combines bilateral bargaining with dynamic innovation to analyze the impact of vertical integration on innovation, pricing and welfare in the chipset and smartphone industries. Using the estimated model, I simulate the counterfactual experiment of a vertical merger, and find that vertical integration increases innovation primarily through the investment effects. The results suggest that the dynamic effect of vertical integration may be large and positive, providing support for giving more weight to this factor in a vertical merger review.

Several simplifying assumptions underlie the model. First, I do not consider vertical integration's effects on the cost primitives. Cost reduction in the case of a successful merger could further increase efficiency. Secondly, I model the pricing game without considering the strategic roles of carriers. This modeling choice is largely motivated by the need to simplify the computation of period profits. The approach is similar to Goettler and Gordon (2011) and Nosko (2014), which focused on the innovation in the CPU industry and abstracted from the role of downstream computer assemblers. An alternative handset-carrier pricing model (e.g. Sinkinson $(2014) ;$ Luo (2016); Fan and Yang (2016)) would imply different estimates of firm profits, but I do not expect the bias to reverse the result: the investment effects are an order of magnitude larger than the price effects in the main specification. Thirdly, I do not consider serially correlated unobserved cost variables, which may be a concern given that the data frequency is monthly. Omitting these variables would bias the estimates of the innovation costs. However, including such a cost component does not change any of the economic argument why innovation increases with a vertical merger. Fourthly, I assume that Qualcomm and handset makers use linear contracts. Linear contracts introduce inefficiency because of double marginalization, but these effects are small compared with the investment effects. 
Table 8: Counterfactual Results: Main Specification, Jan 2009 to Dec 2011

\begin{tabular}{|c|c|c|c|c|}
\hline & & No VI & Investment Coordination & Full VI \\
\hline \multirow{9}{*}{$\begin{array}{l}\text { Innovation Rate: } \\
\quad\left(q_{36}-q_{1}\right) / 35\end{array}$} & Apple & {$[0.0403,0.0444]$} & {$[0.0414,0.0456]$} & {$[0.0414,0.0454]$} \\
\hline & Samsung & {$[0.1073,0.1176]$} & {$[0.1282,0.1305]$} & {$[0.1294,0.1318]$} \\
\hline & HTC & {$[0.0783,0.0828]$} & {$[0.0933,0.0951]$} & {$[0.0939,0.0955]$} \\
\hline & Qualcomm & {$[0.0731,0.0863]$} & {$[0.0972,0.0986]$} & {$[0.0979,0.0999]$} \\
\hline & & & Difference \% & \\
\hline & Apple & & {$[1.65 \%, 2.73 \%]$} & {$[-0.38 \%, 0.00 \%]$} \\
\hline & Samsung & & {$[9.00 \%, 21.60 \%]$} & {$[0.47 \%, 1.00 \%]$} \\
\hline & HTC & & {$[14.13 \%, 19.27 \%]$} & {$[0.12 \%, 0.71 \%]$} \\
\hline & Qualcomm & & {$[12.68 \%, 34.93 \%]$} & {$[0.68 \%, 1.35 \%]$} \\
\hline \multirow{3}{*}{$\begin{array}{c}\text { Consumer Surplus } \\
\text { (\$ Billion) }\end{array}$} & & {$[22.8098,23.5128]$} & {$[24.3182,24.6586]$} & {$[24.6426,24.9367]$} \\
\hline & & & Difference \% & \\
\hline & & & {$[3.52 \%, 6.61 \%]$} & {$[1.02 \%, 1.33 \%]$} \\
\hline \multirow{3}{*}{$\begin{array}{c}\mathrm{CS}+\mathrm{PS} \\
(\$ \text { Billion })\end{array}$} & & {$[47.3067,48.5485]$} & {$[49.8703,50.5868]$} & {$[50.2159,50.8546]$} \\
\hline & & & Difference \% & \\
\hline & & & {$[2.99 \%, 5.42 \%]$} & {$[0.44 \%, 0.69 \%]$} \\
\hline \multirow{9}{*}{$\begin{array}{l}\text { Investment } \\
(\$ \text { Billion })\end{array}$} & Apple & {$[5.0010,5.7514]$} & {$[5.4768,6.1684]$} & {$[5.4438,6.1684]$} \\
\hline & Samsung & {$[1.9861,2.5103]$} & {$[2.3619,2.8610]$} & {$[2.3818,2.8867]$} \\
\hline & HTC & {$[2.8243,3.3867]$} & {$[5.0169,5.1862]$} & {$[5.1319,5.2378]$} \\
\hline & Qualcomm & {$[0.5649,0.6458]$} & {$[0.8673,0.9249]$} & {$[0.8757,0.9370]$} \\
\hline & & & Difference \% & \\
\hline & Apple & & {$[4.90 \%, 10.64 \%]$} & {$[-1.42 \%, 0.00 \%]$} \\
\hline & Samsung & & {$[13.97 \%, 20.24 \%]$} & {$[0.30 \%, 0.90 \%]$} \\
\hline & HTC & & {$[52.52 \%, 77.80 \%]$} & {$[0.33 \%, 2.29 \%]$} \\
\hline & Qualcomm & & {$[36.16 \%, 63.73 \%]$} & {$[0.96 \%, 1.33 \%]$} \\
\hline \multirow{7}{*}{$\begin{array}{l}\text { Producer Surplus } \\
\qquad(\$ \text { Billion })\end{array}$} & Apple & {$[16.2596,16.7123]$} & {$[16.0346,16.5620]$} & {$[15.9860,16.4977]$} \\
\hline & Samsung & {$[5.3042,5.5648]$} & {$[5.7950,6.1004]$} & {$[5.8327,6.1299]$} \\
\hline & HTC+Qualcomm & {$[2.8294,2.9857]$} & {$[3.3428,3.4171]$} & {$[3.3946,3.4574]$} \\
\hline & & & Difference \% & \\
\hline & Apple & & {$[-1.38 \%,-0.14 \%]$} & {$[-0.41 \%,-0.25 \%]$} \\
\hline & Samsung & & {$[4.14 \%, 15.01 \%]$} & {$[-0.19 \%, 0.68 \%]$} \\
\hline & HTC+Qualcomm & & {$[13.99 \%, 18.38 \%]$} & {$[0.86 \%, 1.55 \%]$} \\
\hline \multirow{7}{*}{ Retail Price $(\$)$} & Apple & {$[160.2546,164.0366]$} & {$[160.0248,164.2321]$} & {$[159.8585,163.9179$} \\
\hline & Samsung & {$[224.9629,231.3038]$} & {$[239.6018,245.4518]$} & {$[241.4173,247.1854$} \\
\hline & HTC & {$[198.1648,202.4500]$} & {$[212.1613,213.7752]$} & {$[199.4719,200.7462$} \\
\hline & & & Difference $\%$ & \\
\hline & Apple & & {$[-0.26 \%, 0.39 \%]$} & {$[-0.19 \%,-0.09 \%]$} \\
\hline & Samsung & & {$[3.59 \%, 9.11 \%]$} & {$[0.31 \%, 0.76 \%]$} \\
\hline & HTC & & {$[5.43 \%, 7.22 \%]$} & {$[-6.24 \%,-5.98 \%]$} \\
\hline \multirow{5}{*}{ Chipset Price $(\$)$} & Samsung & {$[31.3554,31.3706]$} & [31.4121, 31.4150] & {$[32.4944,32.5180]$} \\
\hline & HTC & {$[31.3886,31.3990]$} & {$[31.4238,31.4278]$} & - \\
\hline & & & Difference \% & \\
\hline & Samsung & & {$[0.14 \%, 0.19 \%]$} & {$[3.44 \%, 3.51 \%]$} \\
\hline & HTC & & {$[0.09 \%, 0.11 \%]$} & - \\
\hline \multirow{3}{*}{$\begin{array}{l}\text { Proportion of Samsung } \\
\text { Using Qualcomm }\end{array}$} & & {$[0.4473,0.4895]$} & {$[0.5651,0.5889]$} & {$[0.5679,0.5908]$} \\
\hline & & & Difference $\%$ & \\
\hline & & & {$[20.30 \%, 28.24 \%]$} & {$[-0.10 \%, 0.71 \%]$} \\
\hline
\end{tabular}


Table 9: Estimates of Innovation Costs

\begin{tabular}{|c|c|c|c|}
\hline & & \multicolumn{2}{|c|}{ 95\% Confidence Set } \\
\hline & & With Royalties & Additional Handset Makers \\
\hline \multirow{4}{*}{$\gamma_{0}$} & Apple & {$[-0.34,0.65]$} & {$[0.06,0.14]$} \\
\hline & Samsung & {$[-2.54,0.01]$} & {$[1.33,1.52]$} \\
\hline & HTC & {$[-0.61,0.14]$} & {$[-0.12,0.07]$} \\
\hline & Qualcomm & {$[-4.89,-3.58]$} & {$[-4.15,-3.07]$} \\
\hline \multirow{4}{*}{$\gamma_{1}$} & Apple & {$[21.65,25.71]$} & {$[20.46,20.96]$} \\
\hline & Samsung & {$[7.32,13.08]$} & {$[3.49,4.49]$} \\
\hline & HTC & {$[10.61,15.87]$} & {$[10.03,10.03]$} \\
\hline & Qualcomm & {$[14.57,20.20]$} & {$[4.27,7.80]$} \\
\hline$\gamma_{2}$ & Samsung & {$[4.86,5.76]$} & {$[4.52,4.71]$} \\
\hline \multirow{2}{*}{$\sigma$} & Handset & {$[5.39,6.92]$} & {$[5.09,5.77]$} \\
\hline & Qualcomm & {$[0.97,3.08]$} & {$[1.84,2.52]$} \\
\hline
\end{tabular}

I report the min and max of each parameter in the confidence set.

The confidence set consists of a set of vectors of parameters

that satisfy 13 and is not a Cartesian product of the intervals above.

Table 10: Counterfactual Results: Royalty Fees, Jan 2009 to Dec 2011

\begin{tabular}{|c|c|c|c|c|}
\hline & & No VI & Investment Coordination & Full VI \\
\hline \multirow{9}{*}{$\begin{array}{l}\text { Innovation Rate: } \\
\qquad\left(q_{36}-q_{1}\right) / 35\end{array}$} & Apple & {$[0.0359,0.0420]$} & {$[0.0372,0.0435]$} & {$[0.0370,0.0434]$} \\
\hline & Samsung & {$[0.0975,0.1159]$} & {$[0.1144,0.1364]$} & {$[0.1155,0.1384]$} \\
\hline & HTC & {$[0.0712,0.0910]$} & {$[0.0898,0.1059]$} & {$[0.0909,0.1064]$} \\
\hline & Qualcomm & {$[0.0597,0.0792]$} & {$[0.0778,0.1014]$} & {$[0.0789,0.1034]$} \\
\hline & & & Difference \% & \\
\hline & Apple & & {$[2.02 \%, 3.87 \%]$} & {$[-0.81 \%,-0.27 \%]$} \\
\hline & Samsung & & {$[15.02 \%, 21.73 \%]$} & {$[0.62 \%, 1.48 \%]$} \\
\hline & HTC & & {$[15.05 \%, 26.08 \%]$} & {$[0.24 \%, 1.22 \%]$} \\
\hline & Qualcomm & & {$[25.64 \%, 36.83 \%]$} & {$[0.88 \%, 1.94 \%]$} \\
\hline \multirow{3}{*}{$\begin{array}{l}\text { Consumer Surplus } \\
\qquad(\$ \text { Billion })\end{array}$} & & {$[22.1926,24.1541]$} & {$[23.6418,25.8975]$} & {$[24.4723,26.8162]$} \\
\hline & & & Difference \% & \\
\hline & & & {$[6.35 \%, 8.06 \%]$} & {$[3.44 \%, 3.72 \%]$} \\
\hline \multirow{3}{*}{$\begin{array}{c}\text { CS+PS } \\
(\$ \text { Billion })\end{array}$} & & {$[52.1515,56.1937]$} & {$[55.1097,59.5594]$} & {$[56.1202,60.6983]$} \\
\hline & & & Difference \% & \\
\hline & & & {$[5.29 \%, 6.71 \%]$} & {$[1.82 \%, 1.91 \%]$} \\
\hline
\end{tabular}


Table 11: Counterfactual Results: Additional Handset Makers, Jan 2009 to Dec 2011

\begin{tabular}{|c|c|c|c|c|}
\hline & & No VI & Investment Coordination & Full VI \\
\hline \multirow{9}{*}{$\begin{array}{l}\text { Innovation Rate: } \\
\quad\left(q_{36}-q_{1}\right) / 35\end{array}$} & Apple & {$[0.0359,0.0372]$} & {$[0.0364,0.0375]$} & {$[0.0363,0.0374]$} \\
\hline & Samsung & {$[0.0979,0.1064]$} & {$[0.1072,0.1147]$} & {$[0.1076,0.1152]$} \\
\hline & HTC & {$[0.0666,0.0674]$} & {$[0.0685,0.0704]$} & {$[0.0686,0.0705]$} \\
\hline & Qualcomm & {$[0.0638,0.0737]$} & {$[0.0742,0.0828]$} & {$[0.0747,0.0831]$} \\
\hline & & & Difference \% & \\
\hline & Apple & & {$[0.78 \%, 1.37 \%]$} & {$[-0.32 \%,-0.08 \%]$} \\
\hline & Samsung & & {$[7.80 \%, 9.55 \%]$} & {$[0.35 \%, 0.51 \%]$} \\
\hline & HTC & & {$[2.06 \%, 5.69 \%]$} & {$[0.00 \%, 0.16 \%]$} \\
\hline & Qualcomm & & {$[12.33 \%, 16.23 \%]$} & {$[0.42 \%, 0.76 \%]$} \\
\hline \multirow{3}{*}{$\begin{array}{c}\text { Consumer Surplus } \\
\text { (\$ Billion) }\end{array}$} & & {$[29.1741,29.8368]$} & {$[29.7669,30.4251]$} & {$[29.9440,30.6030]$} \\
\hline & & & Difference \% & \\
\hline & & & {$[1.97 \%, 2.31 \%]$} & {$[0.58 \%, 0.62 \%]$} \\
\hline \multirow{3}{*}{$\begin{array}{c}\text { CS+PS } \\
(\$ \text { Billion })\end{array}$} & & {$[56.5889,57.5216]$} & {$[57.4451,58.3713]$} & {$[57.5912,58.5211]$} \\
\hline & & & Difference \% & \\
\hline & & & {$[1.48 \%, 1.76 \%]$} & {$[0.25 \%, 0.27 \%]$} \\
\hline
\end{tabular}

\section{References}

Aghion, Philippe and Richard Holden, "Incomplete contracts and the theory of the firm: What have we learned over the past 25 years?," The Journal of Economic Perspectives, 2011, 25 (2), 181-197.

_, Nick Bloom, Richard Blundell, Rachel Griffith, and Peter Howitt, "Competition and innovation: An inverted-U relationship," The Quarterly Journal of Economics, 2005, 120 (2), $701-728$.

Allain, Marie-Laure, Claire Chambolle, and Patrick Rey, "Vertical integration, innovation and foreclosure," 2011.

Andrews, Donald WK and Gustavo Soares, "Inference for parameters defined by moment inequalities using generalized moment selection," Econometrica, 2010, 78 (1), 119-157.

Arghire, Ionut, "Samsung Pays 1.3Billionfor PatentLicensestoQualcomm," softpedia.com, 2009.

Asker, John, "Diagnosing Foreclosure due to Exclusive Dealing," Journal of Industrial Economics, forthcoming, 2015.

Atalay, Enghin, Ali Hortaçsu, and Chad Syverson, "Vertical integration and input flows," The American Economic Review, 2014, 104 (4), 1120-1148. 
Baker, George, Robert Gibbons, and Kevin J Murphy, "Relational Contracts and the Theory of the Firm," The Quarterly Journal of Economics, 2002, 117 (1), 39-84.

Berry, Steven, James Levinsohn, and Ariel Pakes, "Automobile prices in market equilibrium," Econometrica: Journal of the Econometric Society, 1995, pp. 841-890.

Berry, Steven T and Philip A Haile, "Identification in differentiated products markets using market level data," Econometrica, 2014, 82 (5), 1749-1797.

Bonnet, Céline and Pierre Dubois, "Inference on vertical contracts between manufacturers and retailers allowing for nonlinear pricing and resale price maintenance," The RAND Journal of Economics, 2010, 41 (1), 139-164.

Borkovsky, R, "The timing of version releases: A dynamic duopoly model," 2012.

Bradshaw, Tim, "Apple in-house chip design unit gives it smartphone edge," www.ft.com, 2015.

Brenkers, Randy and Frank Verboven, "Liberalizing a distribution system: the European car market," Journal of the European Economic Association, 2006, 4 (1), 216-251.

Bresnahan, Timothy F, "The oligopoly solution concept is identified," Economics Letters, 1982, $10(1-2), 87-92$.

Che, Yeon-Koo and József Sákovics, "A dynamic theory of holdup," Econometrica, 2004, 72 (4), 1063-1103.

_ and _ , "Contractual remedies to the hold-up problem: A dynamic perspective," in "American Law \& Economics Association Annual Meetings" bepress 2007, p. 14.

Chen, Dong and David Waterman, "Vertical ownership, program network carriage, and tier positioning in cable television: An empirical study," Review of Industrial Organization, 2007, 30 (3), 227-251.

Chen, Xiang, Yiran Chen, Zhan Ma, and Felix CA Fernandes, "How is energy consumed in smartphone display applications?," in "Proceedings of the 14th Workshop on Mobile Computing Systems and Applications" ACM 2013, p. 3.

Cheng, Francisco, "What Integrating a 4G LTE Modem means to the Snapdragon S4 Processor and Battery life," qualcomm.com, 2012.

Chernozhukov, Victor, Han Hong, and Elie Tamer, "Estimation and confidence regions for parameter sets in econometric models," Econometrica, 2007, 75 (5), 1243-1284.

Chipty, Tasneem, "Vertical integration, market foreclosure, and consumer welfare in the cable television industry," American Economic Review, 2001, pp. 428-453.

Clark, Don, "Does Apple Enjoy a Licensing Loophole on iPhone?," wsj.com, 2009. 
Colon, Alex, "Tests show iPhone 5s A7 chip is dual-core, still beats quad-core Android competitors," gigaom.com, 2013.

Crawford, Gregory S and Ali Yurukoglu, "The Welfare Effects of Bundling in Multichannel Television Markets," The American Economic Review, 2012, 102 (2), 643-685.

_, Robin S Lee, Michael D Whinston, and Ali Yurukoglu, "The Welfare Effects of Vertical Integration in Multichannel Television Markets," 2015.

CSIMarket, "Apple (AAPL) Sales per Country and Region, Sep.27.2014 Annual Report," CSIMarket.com, 2014.

Draganska, Michaela, Daniel Klapper, and Sofia B Villas-Boas, "A larger slice or a larger pie? An empirical investigation of bargaining power in the distribution channel," Marketing Science, 2010, 29 (1), 57-74.

Echenique, Federico and Ivana Komunjer, "Testing models with multiple equilibria by quantile methods," California Institute of Technology Social Science Working Paper, 2007, 1244.

Egesdal, Michael, Zhenyu Lai, and Che-Lin Su, "Estimating dynamic discrete-choice games of incomplete information," Quantitative Economics, 2015, 6 (3), 567-597.

Eizenberg, Alon, "Upstream innovation and product variety in the us home pc market," The Review of Economic Studies, 2014, 81 (3), 1003-1045.

Ericson, Richard and Ariel Pakes, "Markov-perfect industry dynamics: A framework for empirical work," The Review of Economic Studies, 1995, 62 (1), 53-82.

Fan, Ying and Chenyu Yang, "Competition, Product Proliferation and Welfare: A Study of the US Smartphone Market,” 2016.

Gartner, "Market Share: Mobile Devices by Region and Country, 4Q11 and 2011," 2012.

Geradin, Damien, Anne Layne-Farrar, and Nicolas Petit, EU competition law and economics, Oxford University Press, 2012.

Goettler, Ronald L and Brett R Gordon, "Does AMD spur Intel to innovate more?," Journal of Political Economy, 2011, 119 (6), 1141-1200.

Gowrisankaran, Gautam, Aviv Nevo, and Robert Town, "Mergers when prices are negotiated: Evidence from the hospital industry," The American Economic Review, 2014, 105 (1), 172-203.

Grennan, Matthew, "Price Discrimination and Bargaining: Empirical Evidence from Medical Devices," The American Economic Review, 2013, 103 (1), 145.

Grossman, Sanford J and Oliver D Hart, "The Costs and Benefits of Ownership: A Theory of Vertical and Lateral Integration," The Journal of Political Economy, 1986, pp. 691-719. 
Halonen, Maija, "Reputation and the Allocation of Ownership," The Economic Journal, 2002, 112 (481), 539-558.

Hart, Oliver and John Moore, "Property Rights and the Nature of the Firm," Journal of political economy, 1990, pp. 1119-1158.

Hastings, Justine S, "Vertical Relationships and Competition in Retail Gasoline Markets: Empirical Evidence from Contract Changes in Southern California," American Economic Review, 2004, pp. $317-328$.

- and Richard J Gilbert, "Market Power, Vertical Integration and the Wholesale Price of Gasoline*," The Journal of Industrial Economics, 2005, 53 (4), 469-492.

Ho, Kate and Robin S Lee, "Insurer Competition in Health Care Markets," Econometrica, conditionally accepted, 2016.

Holmström, Bengt and John Roberts, "The boundaries of the firm revisited," The Journal of Economic Perspectives, 1998, 12 (4), 73-94.

Horn, Henrick and Asher Wolinsky, "Bilateral monopolies and incentives for merger," The RAND Journal of Economics, 1988, pp. 408-419.

Hortacsu, Ali and Chad Syverson, "Cementing relationships: Vertical integration, foreclosure, productivity, and prices," Journal of political economy, 2007, 115 (2), 250-301.

Igami, Mitsuru, "Estimating the Innovator's Dilemma: Structural Analysis of Creative Destruction in the Hard Disk Drive Industry, 1981-1998," Journal of Political Economy, forthcoming, 2015.

Lafontaine, Francine and Margaret Slade, "Vertical integration and firm boundaries: the evidence," Journal of Economic Literature, 2007, 45 (3), 629-685.

Low, Cherlynn, "Xiaomi is reportedly building its own phone processor," http://wccftech.com, 2017.

Luo, Rong, "The Operating System Network Effect and Carriers Dynamic Pricing of Smartphones," 2016.

Mock, Dave, The qualcomm equation: how a fledgling telecom company forged a new path to big profits and market dominance, AMACOM Div American Mgmt Assn, 2005.

Mortimer, Julie H, "Vertical contracts in the video rental industry," The Review of Economic Studies, 2008, 75 (1), 165-199.

Murry, Charles, "Advertising in Vertical Relationships: An Equilibrium Model of the Automobile Industry," 2015.

Natividad, Gabriel, "Integration and productivity: Satellite-tracked evidence," Management Science, 2014, 60 (7), 1698-1718. 
Nosko, Chris, "Competition and Quality Choice in the CPU Market," 2014.

Perry, Martin K, "Vertical integration: Determinants and effects," Handbook of Industrial Organization, 1989, 1, 183-255.

Phone Arena, "We changed the LG G3's display resolution to 1080p - we got superb performance and negligible battery life increases," phonearena.com, 2015.

Riordan, Michael H, "Competitive effects of vertical integration," Handbook of antitrust economics, 2008, 14582.

Romano, Joseph P, Azeem M Shaikh, and Michael Wolf, "A Practical Two-Step Method for Testing Moment Inequalities," Econometrica, 2014, 82 (5), 1979-2002.

Savov, Vlad, "This is Qualcomm's world and we're all just living in it," theverge.com, 2014.

Shi, Xiaoxia and Matthew Shum, "Simple two-stage inference for a class of partially identified models," Econometric Theory, 2015, 31 (03), 493-520.

Sinkinson, Michael, "Pricing and entry incentives with exclusive contracts: Evidence from smartphones," Available at SSRN 2391745, 2014.

Smith, Matt, "Superior silicon: How Apple is beating chipmakers at their own game," http://www.digitaltrends.com, 2015.

Sohail, Omar, "Sony And LG Have Been Reported To Develop Their Own Smartphone Chipsets," http://wccftech.com, 2015.

Tirole, Jean, "Incomplete contracts: Where do we stand?," Econometrica, 1999, 67 (4), 741-781.

Villas-Boas, Sofia Berto, "Vertical relationships between manufacturers and retailers: Inference with limited data," The Review of Economic Studies, 2007, 74 (2), 625-652.

Waterman, David and Andrew A Weiss, "The Effects of Vertical Integration between Cable Television Systems and Pay Cable Networks," Journal of Econometrics, 1996, 72 (1), 357-395.

Wollmann, Thomas, "Trucks without Bailouts: Equilibrium Product Characteristics for Commercial Vehicles," 2016.

Woyke, Elizabeth, The Smartphone: Anatomy of an Industry, The New Press, 2014.

Yang, Da, Chock Gan, PR Chidambaram, Giri Nallapadi, John Zhu, SC Song, Jeff $\mathrm{Xu}$, and Geoffrey Yeap, "Technology-design-manufacturing co-optimization for advanced mobile SoCs," in "SPIE Advanced Lithography" International Society for Optics and Photonics 2014, pp. $90530 \mathrm{~N}-90530 \mathrm{~N}$. 
Yeap, Geoffrey, "Smart mobile SoCs driving the semiconductor industry: Technology trend, challenges and opportunities," in "2013 IEEE International Electron Devices Meeting” IEEE 2013, pp. $1-3$.

\section{Appendix}

\section{A First Order Conditions in the Static Pricing Game}

I omit the time subscript. Qualcomm and handset maker $n$ bargain over $\psi$. Handset maker $n$ 's profit at the point of disagreement is

$$
\tilde{\pi}^{n}=\sum_{j \in \mathcal{J}_{n} \cap \mathcal{J}_{Q}}\left(\tilde{p}_{j}-\omega_{j}-\bar{\psi}\right) \tilde{D}_{j}+\sum_{j \in \mathcal{J}_{n} \backslash \mathcal{J}_{Q}}\left(\tilde{p}_{j}-\omega_{j}\right) \tilde{D}_{j}
$$

and Qualcomm's disagreement profit is

$$
\tilde{\pi}^{Q}=\sum_{j \in \mathcal{J}_{n} \backslash \mathcal{J}_{Q}}\left(\psi_{j}-\underline{\psi}\right) \tilde{D}_{j}
$$

where $\sim$ denotes the recalculated equilibrium quantities at the point of disagreement.

The first order condition of the bargaining game is

$$
\psi=\underline{\psi}+\Theta^{-1} \Phi
$$

where $\Theta$ and $\Phi$ are given by the following:

$$
\begin{gathered}
\Theta=d \Pi+d \Gamma, \\
\Phi=-\left(s_{Q}+d \Omega\right),
\end{gathered}
$$

where in vector and matrix notation,

$$
d \Pi=\nabla D_{p} \nabla p_{\psi} * L^{Q},
$$

where $L^{Q}$ is a binary matrix such that $L_{i, j}^{Q}=1$ if $i, j$ both use Qualcomm chipsets, and 0 otherwise, and

$$
d \Gamma=\left(\begin{array}{ccc}
\frac{\partial \pi^{n=1}}{\partial \psi_{n=1}} & & \\
& \ddots & \\
& & \frac{\partial \pi^{n=N}}{\partial \psi_{n=N}^{c}}
\end{array}\right)\left(\begin{array}{cc}
\left|\mathcal{J}_{Q} \cap \mathcal{J}_{n=1}\right| \text { replications } & \left\{\frac{\boldsymbol{D}_{Q}-\tilde{\boldsymbol{D}}_{Q}(n=1) \cdot \imath_{n=1}}{\pi^{n=1}-\tilde{\pi}^{n=1}}\right. \\
\vdots \\
\left|\mathcal{J}_{Q} \cap \mathcal{J}_{n=N}\right| \text { replications }\left\{\frac{\boldsymbol{D}_{Q}-\tilde{\boldsymbol{D}}_{Q}(n=N) \cdot \iota_{n=N}}{\pi^{n=N}-\tilde{\pi}^{n=N}}\right.
\end{array}\right),
$$

where $\frac{\partial \pi_{n}}{\partial \psi_{n}}$ is a block of diagonal matrix, the derivative of handset maker $n$ 's profit with respect to 
the price of each of its Qualcomm chipset:

$$
\frac{\partial \pi_{n}}{\partial \psi_{i}}=\sum_{j \in \mathcal{J}_{n t}} \frac{\partial p_{j}}{\partial \psi_{i}} D_{j}-D_{i}+\sum_{j \in \mathcal{J}_{n t}}\left(p_{j}-\omega_{j}-\psi_{j}\right) \sum_{k} \frac{\partial D_{j}}{\partial p_{k}} \frac{\partial p_{k}}{\partial \psi_{i}} .
$$

and $\tilde{\boldsymbol{D}}_{Q}(n)$ corresponds with the vector of demand for Qualcomm chipsets at the disagreement point in the Qualcomm- $n$ bargaining pair. $\imath_{n}$ is a row vector of binaries corresponding with each product, and equal to 0 if corresponding with firm $n$ 's products.

When Qualcomm is integrated with $\breve{n}$, the FOC's of the bargaining equilibrium becomes

$$
\psi=\underline{\psi}+\Theta^{-1} \breve{\Phi}
$$

where $\breve{\Phi}=-\left(D_{Q}+d \Lambda+d \Omega\right)$, and

$$
d \Omega=\left(\begin{array}{ccc}
\frac{\partial \pi_{n=1}}{\partial \psi_{n=1}} & \\
& \ddots & \\
& & \frac{\partial \pi_{n=N}}{\partial \psi_{n=N}}
\end{array}\right) \cdot\left(\begin{array}{c}
\left|\mathcal{J}_{Q} \cap \mathcal{J}_{n=1}\right| \text { replications }\left\{\frac{\boldsymbol{p}_{\breve{n}}}{\partial \psi}+\left[\boldsymbol{p}_{\breve{n}}-\boldsymbol{\omega}_{\breve{n}}-\boldsymbol{\psi}_{\breve{n}}\right] \nabla D_{p} \tilde{\pi}^{\breve{n}}(n=1)\right. \\
\pi^{n=1}-\tilde{\pi}^{n=1} \\
\vdots \\
\left|\mathcal{J}_{Q} \cap \mathcal{J}_{n=N}\right| \text { replications }\left\{\frac{\pi^{\breve{n}}-\tilde{\pi}^{\check{n}}(n=N)}{\pi^{n=N}-\tilde{\pi}^{n=N}}\right.
\end{array}\right),
$$

where $\tilde{\pi}^{\breve{n}}(n)$ corresponds with $\breve{n}$ 's profit at the disagreement point of Qualcomm- $n$ pair. In addition, the integrated Qualcomm would only negotiate chipset prices with non-integrated downstream rivals.

\section{B Product Set Simulation}

In the specification that includes just Apple, HTC and Samsung, I sample from the empirical distribution of product sets in data to compute the expected profits. I illustrate how I compute the period profits under the state of handset makers $\left(q^{A p p l e}, q^{H T C}, q^{S a m}, \eta^{S a m}\right)$ in period $t$. I start with Apple. I first uniformly sample $\hat{t}_{A}$, from $1 \ldots 51$. I adjust the quality of products in $J_{A, \hat{t}_{A}}$ by

$$
q_{j \hat{t}_{A}}-\max _{k \in \mathcal{J}_{A, \hat{t}_{A}}} q_{k}+q^{\text {Apple }}
$$

Call this new set $\hat{J}_{A}$. I repeat this process, by sampling $\hat{t}_{H}$ for HTC, adjust qualities similarly and denote the resulting set of products as $\hat{J}_{H}$. Given Samsung frontier $q^{\text {Sam }}$ and $\eta^{\text {Sam }} \in\{0.3,0.5,0.7\}$, I first collect all periods where the proportion of Samsung handsets using Qualcomm chipsets is closest to $\eta^{\text {Sam }}$. Denote the set as $\mathcal{T}_{\eta^{\text {Sam }}}$. I then sample one period $\hat{t}_{S}$ from $\mathcal{T}_{\eta^{\text {Sam }}}$ and construct $\hat{J}_{S}$. I pair the set $\hat{J}_{A} \cup \hat{J}_{H} \cup \hat{J}_{S}$ with appropriate time fixed effects in $t$ and sample from the empirical distribution of demand and marginal cost shocks and bargaining weights to calculate period profits for Qualcomm and downstream handset makers. I use the average of profits from 50 draws of 
product sets and shocks as the period profits in the dynamic game.

In the specification that includes $\tilde{\mathcal{N}}=\{$ BlackBerry, Motorla, LG $\}$, I sample from the empirical distribution of product sets of $n \in \tilde{\mathcal{N}}$ conditional on HTC and Samsung frontier. Specifically, in every period $t$ in data, denote the HTC and Samsung frontiers, as defined in the main text, as $\bar{q}_{t}=\max \left\{q_{t}^{H T C}, q_{t}^{S a m}\right\}$ and the frontier of $n$ 's products as $\bar{q}_{t}^{n}$. I first compute the average distance $\Delta q_{n}=\sum_{t}\left(\bar{q}_{t}-\bar{q}_{n t}\right) / T$. To calculate the period profit corresponding with the state $\left(q^{\text {Apple }}, q^{H T C}, q^{S a m}, \eta^{S a m}\right)$ in a period, I first sample Apple, HTC and Samsung's product sets as described above. I then randomly sample a period $\hat{t}$. I adjust the quality $q_{j \hat{t}}$ of $j$ in $J_{n \hat{t}}$ to

$$
q_{j \hat{t}}-\max _{k \in \mathcal{J}_{n \hat{t}}} q_{k}+\max \left\{q^{H T C}, q^{S a m}\right\}-\Delta q_{n}
$$

and obtain $\hat{J}_{n}$. I repeat this process for every $n \in \tilde{\mathcal{N}}$ and calculate period profits for all upstream and downstream firms based on $\hat{J}_{A} \cup \hat{J}_{H} \cup \hat{J}_{S} \cup_{n \in \tilde{\mathcal{N}}} \hat{J}_{n}$, a draw of shocks and a draw of the bargaining weight. The expected profit is calculated as the average of period profits based on 50 draws.

\section{Solving, Estimating and Simulating the Dynamic Model}

I set the quality increment for Qualcomm to be $\Delta=0.25$, and $a^{Q} \in\{0, \Delta, 2 \Delta, \ldots, 6 \Delta\}$. The handset makers' quality increment is $\delta=0.25$, with $a_{q}^{n} \in\{0, \delta, 2 \delta, 3 \delta\}$ and $a_{\eta}^{\text {Samsung }} \in\{30 \%, 50 \%, 70 \%\}$. The specification matches most of the actions observed in data. Because of the constraint that Samsung and HTC qualities do not exceed Qualcomm's quality, I track the difference between Qualcomm and the maximum of HTC and Samsung's quality frontiers, $\delta^{Q}=q^{Q}-\max \left\{q^{\text {Samsung }}, q^{H T C}\right\} \geq$ 0 , instead of Qualcomm quality frontier directly, in addition to handset makers' quality frontiers and Samsung's proportion of handsets using Qualcomm chipsets. The value function is parameterized as a third degree complete polynomial of Apple, Samsung and HTC's quality levels. To precisely calculate the value function given $\delta^{Q}, \eta$ and $t$, I compute a different set of polynomial coefficients specific to each combination of $\left\{t, \eta, \delta^{Q}\right\}$, where $t=1, \ldots, T, \eta \in\{30 \%, 50 \%, 70 \%\}$, and $\delta^{Q} \in\{0, \delta, \ldots, 10 \delta\}$. I solve the value functions at the zeros of the Chebyshev polynomials and interpolate the value functions at other states. The choice probabilities of each firm are simulated with 200 draws of investment cost shocks.

In the formulation of Shi and Shum (2015), data do not directly enter the inequality constraint. Instead, the inequality constraint (14) is converted into an equality constraint by introducing a slackness parameter and adding an inequality constraint that the slackness parameter is positive.

To construct the confidence set in (13), I use a genetic algorithm that searches through an 12-dimensional space with a wide initial range. Each generation of the genetic algorithm iteration has 32 seeds, and I iterate over 96 generations. The intermediate functional values are saved and included in the confidence set if the corresponding $g^{e} / W g^{e}$ is below the critical value. I eventually obtain 300 to 600 points in the confidence set for every specification.

Because the moments I choose are stationary, I use bootstrap to calculate the weighting matrix from data. I block bootstrap consecutive 12-month periods and compute the co-variance matrix of 
the equality moments. $W$ is the inverse of this co-variance matrix.

Qualcomm quality is an unobserved state variable. To deal with the initial value problem, I calibrate the starting value of Qualcomm state and conduct robustness checks. The main specification starts the simulation that Qualcomm is 0.25 below the bound in period 1 . The robustness checks in Appendix $\mathrm{D}$ considers two different starting states for Qualcomm.

Because Qualcomm bounds are based on the quality of handsets using the next generation's chipsets, and the last generation is S4 in data, there is also a "terminal value problem" that there are no quality measures in data to bound Qualcomm quality when it is in generation S4. The first handset using the next generation Qualcomm chipset Snapdragon 600 is Galaxy S4. To construct the quality index for such a phone, I need to calibrate the chipset generation fixed effect. I choose 2.474 for the chipset effect, which is 0.8 larger than the S4 chipset generation effect in demand estimates. The incremental increase in the chipset effect in previous generations is less than 0.63. In choosing a large chipset fixed effect and hence a high upper bound for Qualcomm, I err on the side of understating the benefit of vertical integration.

I stratify points in the confidence set and sample points from each stratum to conduct counterfactual simulations. In principle, I can approximate the confidence set of the counterfactual predictions by using every point in the confidence set to simulate counterfactual scenarios, but this is computationally infeasible. The purpose of the stratified sampling is to obtain a representative set of parameters from the confidence set. Specifically, I first find the centroid of the confidence set given the distance measure $\|\cdot\|_{1}$. Next, I classify all points in the confidence set into 5 groups based on the point's distance to the centroid. Denote the longest distance as $\ell$, group $n$ consists of points

whose distance to the centroid is between $\frac{n-1}{5} \ell$ and $\frac{n}{5} \ell$, inclusive of $\frac{n}{5} \ell$. I then randomly sample 2 points from each group and simulate each counterfactual analysis with a total of 10 points in the confidence set. In Section 6, every sampled point is used to simulate each scenario 240 times. Increasing the number of strata to 6 and the number of sampled points to 24 do not significantly change the result.

\section{Additional Robustness Checks}

I conduct three types of robustness checks in this section. First, I re-estimate the static and/or the dynamic model and compute counterfactuals for 7 different deviations to the assumptions in the main text. Secondly, I examine to what extent the model can rationalize the data when the dynamic incentives are significantly weakened, by estimating the dynamic model when the discount rate is set to be 0.5 . Lastly, I increase the attractiveness of the alternative chipset by decreasing $\bar{\psi}$ and compute the vertical integration counterfactual.

The 7 deviations to the assumptions in the main text are as follows:

1. Potential quality change at the disagreement point. I further allow the handset quality to decrease by 0.3 at the disagreement point.

2. The gross margin may overstate the actual Qualcomm chipset markup. I use $0.9 \times$ observed 
margin to estimate the chipset pricing model.

3. The gross margin may understate the actual Qualcomm chipset markup. I use $1.1 \times$ observed margin to estimate the chipset pricing model.

4. The assumption of the finite horizon. I extend the game to end 12 months after the end of the sample. The time fixed effects in the demand function from $T+1$ to $T+12$ are extrapolated from the demand estimates in earlier periods.

5. The assumption of the sequential move. I assume that the firms move in the alternative order of Qualcomm, HTC, Samsung and Apple.

6. The initial state value of Qualcomm. I assume that the initial Qualcomm quality is 0.75 quality unit below its bound.

7. The initial state value of Qualcomm. I assume that the initial Qualcomm quality is 0.50 quality unit below its bound.

Table 12 reports the estimates in the robustness checks above, in addition to the estimates when the discount factor is set to 0.5. The estimates of the first seven checks are similar to Table 6. Tables 13 through (19) report the counterfactual results. All but one are consistent with the summary in Section 6. In the third exercise, the range of Samsung and Qualcomm innovation rates based on the sampled points are not all greater than 0 , and neither is the (unreported) consumer and overall welfare measure. The increase in HTC innovation is still robust.

When the discount rate is lowered in the last column of Table 12 , I obtain the implausible result that increasing Qualcomm quality actually decreases innovation costs. The exercises suggests that dynamic incentives are important in rationalizing the data.

I also consider the possibility that additional chipset producers may enter and compete with Qualcomm, if Qualcomm is integrated with HTC. I model this possibility as a decrease in $\bar{\psi}$. Table 20 reports the results when $\bar{\psi}$ is decreased by $10 \%$. The pattern of increased innovation in the event of VI is robust. 
Table 12: Estimates of Innovation Costs : 95\% Confidence Set

\begin{tabular}{|c|c|c|c|c|c|c|c|c|c|}
\hline & & (1) & (2) & (3) & (4) & $(5)$ & (6) & (7) & $\beta=0.5$ \\
\hline \multirow{4}{*}{$\gamma_{0}$} & Apple & {$[-0.25,0.16]$} & {$[-0.34,0.92]$} & {$[-0.23,0.53]$} & {$[-0.43,-0.11]$} & {$[-0.50,-0.41]$} & {$[0.53,0.71]$} & {$[-0.07,1.25]$} & {$[-0.67,0.98]$} \\
\hline & Samsung & {$[-0.10,0.67]$} & {$[0.94,1.19]$} & {$[0.14,0.89]$} & {$[0.22,1.52]$} & {$[0.32,1.83]$} & {$[0.11,1.95]$} & {$[0.02,0.55]$} & {$[-0.43,-0.18]$} \\
\hline & HTC & {$[-1.06,-0.06]$} & {$[-0.94,0.31]$} & {$[-0.59,0.19]$} & {$[-2.01,0.39]$} & {$[-0.13,0.50]$} & {$[-0.29,0.89]$} & {$[0.18,1.85]$} & {$[-0.38,-0.01]$} \\
\hline & Qualcomm & {$[-3.95,-2.95]$} & {$[-4.10,-3.80]$} & {$[-4.21,-3.85]$} & {$[-3.76,-2.31]$} & {$[-3.97,-3.72]$} & {$[-3.91,-3.15]$} & {$[-3.94,-3.66]$} & {$[-2.89,-2.89]$} \\
\hline \multirow{4}{*}{$\gamma_{1}$} & Apple & {$[20.13,22.11]$} & {$[19.04,22.84]$} & {$[19.88,23.17]$} & {$[19.24,20.25]$} & {$[25.27,26.74]$} & {$[27.88,29.13]$} & {$[21.92,27.68]$} & {$[29.92,34.11]$} \\
\hline & Samsung & {$[10.47,11.44]$} & {$[8.01,12.01]$} & {$[8.70,11.19]$} & {$[6.28,8.27]$} & {$[10.60,11.21]$} & {$[10.18,15.87]$} & {$[10.71,15.71]$} & {$[15.07,15.07]$} \\
\hline & HTC & {$[10.69,13.00]$} & {$[9.59,13.59]$} & {$[10.08,12.87]$} & {$[9.61,12.27]$} & {$[11.63,12.51]$} & {$[11.10,12.10]$} & {$[9.40,12.83]$} & {$[14.44,15.32]$} \\
\hline & Qualcomm & {$[5.42,6.57]$} & {$[10.19,12.09]$} & {$[4.28,4.90]$} & {$[10.10,23.03]$} & {$[4.41,4.76]$} & {$[4.41,4.84]$} & {$[4.42,6.80]$} & {$[-5.65,-5.03]$} \\
\hline$\gamma_{2}$ & Samsung & {$[4.56,5.63]$} & {$[5.28,6.84]$} & {$[4.67,5.42]$} & {$[4.94,7.71]$} & {$[4.52,5.42]$} & {$[5.27,5.69]$} & {$[4.71,7.60]$} & {$[2.95,3.52]$} \\
\hline \multirow{2}{*}{$\sigma$} & Handset & {$[4.70,5.33]$} & {$[5.08,5.76]$} & {$[5.09,5.88]$} & {$[4.80,5.44]$} & {$[5.74,6.30]$} & {$[7.13,7.54]$} & {$[6.36,7.21]$} & {$[10.80,10.80]$} \\
\hline & Qualcomm & {$[0.56,1.92]$} & {$[2.25,3.71]$} & {$[0.59,0.73]$} & {$[3.03,8.78]$} & {$[0.80,0.98]$} & {$[0.78,1.71]$} & {$[1.71,2.19]$} & {$[2.07,2.66]$} \\
\hline
\end{tabular}

I report the min and max of each parameter in the confidence set. The confidence set consists of a set of vectors of parameters that satisfy 13 and is not a Cartesian product of the intervals above.

Table 13: Counterfactual Result, Robustness Check 1

\begin{tabular}{ccccc}
\hline & & No VI & Investment Coordination & Full VI \\
\hline \hline & Apple & {$[0.0417,0.0422]$} & {$[0.0430,0.0433]$} & {$[0.0429,0.0432]$} \\
& Samsung & {$[0.1023,0.1198]$} & {$[0.1130,0.1206]$} & {$[0.1146,0.1221]$} \\
& HTC & {$[0.0831,0.0884]$} & {$[0.0936,0.0951]$} & {$[0.0950,0.0960]$} \\
Innovation Rate: & Qualcomm & {$[0.0666,0.0863]$} & {$[0.0785,0.0868]$} & {$[0.0803,0.0885]$} \\
\cline { 2 - 5 }$\left(q_{36}-q_{1}\right) / 35$ & & Difference \% & \\
\cline { 2 - 5 } & Apple & {$[2.54 \%, 3.40 \%]$} & {$[-0.47 \%,-0.20 \%]$} \\
& Samsung & {$[-0.65 \%, 12.41 \%]$} & {$[0.86 \%, 1.49 \%]$} \\
& HTC & {$[7.36 \%, 13.26 \%]$} & {$[0.83 \%, 1.45 \%]$} \\
& Qualcomm & {$[-1.64 \%, 22.39 \%]$} & {$[1.35 \%, 2.28 \%]$} \\
\hline
\end{tabular}


Table 14: Counterfactual Result, Robustness Check 2

\begin{tabular}{ccccc}
\hline & & No VI & Investment Coordination & Full VI \\
\hline \hline & Apple & {$[0.0406,0.0427]$} & {$[0.0417,0.0436]$} & {$[0.0416,0.0436]$} \\
& Samsung & {$[0.1037,0.1125]$} & {$[0.1454,0.1479]$} & {$[0.1458,0.1488]$} \\
& HTC & {$[0.0833,0.0888]$} & {$[0.0974,0.0986]$} & {$[0.0976,0.0986]$} \\
Innovation Rate: & Qualcomm & {$[0.0681,0.0779]$} & {$[0.1138,0.1177]$} & {$[0.1143,0.1186]$} \\
\cline { 2 - 5 }$\left(q_{36}-q_{1}\right) / 35$ & & & Difference \% & \\
\cline { 2 - 5 } & Apple & {$[2.17 \%, 2.78 \%]$} & {$[-0.28 \%, 0.00 \%]$} \\
& Samsung & {$[31.16 \%, 40.23 \%]$} & {$[0.28 \%, 0.61 \%]$} \\
& HTC & {$[10.98 \%, 16.95 \%]$} & {$[0.03 \%, 0.18 \%]$} \\
& Qualcomm & {$[51.06 \%, 67.08 \%]$} & {$[0.43 \%, 0.79 \%]$} \\
\hline
\end{tabular}

Table 15: Counterfactual Result, Robustness Check 3

\begin{tabular}{ccccc}
\hline & & No VI & Investment Coordination & Full VI \\
\hline \hline & Apple & {$[0.0371,0.0464]$} & {$[0.0386,0.0472]$} & {$[0.0385,0.0472]$} \\
& Samsung & {$[0.1042,0.1153]$} & {$[0.1067,0.1082]$} & {$[0.1070,0.1085]$} \\
& HTC & {$[0.0834,0.0889]$} & {$[0.0937,0.0947]$} & {$[0.0939,0.0951]$} \\
Innovation Rate: & Qualcomm & {$[0.0692,0.0838]$} & {$[0.0732,0.0743]$} & {$[0.0737,0.0749]$} \\
\cline { 2 - 5 }$\left(q_{36}-q_{1}\right) / 35$ & & & {$[1.32 \%, 4.06 \%]$} & {$[-0.26 \%,-0.06 \%]$} \\
& Apple & {$[-7.53 \%, 3.30 \%]$} & {$[0.24 \%, 0.43 \%]$} \\
& Samsung & & {$[6.09 \%, 12.69 \%]$} & {$[0.12 \%, 0.43 \%]$} \\
& HTC & & {$[-11.67 \%, 6.73 \%]$} & {$[0.59 \%, 0.91 \%]$} \\
\hline
\end{tabular}

Table 16: Counterfactual Result, Robustness Check 4

\begin{tabular}{ccccc}
\hline & & No VI & Investment Coordination & Full VI \\
\hline \hline & Apple & {$[0.0394,0.0495]$} & {$[0.0400,0.0502]$} & {$[0.0399,0.0501]$} \\
& Samsung & {$[0.0928,0.1091]$} & {$[0.1290,0.1587]$} & {$[0.1297,0.1594]$} \\
& HTC & {$[0.0647,0.0940]$} & {$[0.0858,0.1193]$} & {$[0.0865,0.1195]$} \\
Innovation Rate: & Qualcomm & {$[0.0569,0.0737]$} & {$[0.0989,0.1271]$} & {$[0.0994,0.1277]$} \\
\cline { 2 - 5 }$\left(q_{36}-q_{1}\right) / 35$ & & & {$[0.74 \%, 1.97 \%]$} & {$[-0.23 \%, 0.00 \%]$} \\
\cline { 2 - 5 } & Apple & {$[18.16 \%, 49.86 \%]$} & {$[0.41 \%, 0.78 \%]$} \\
& Samsung & & {$[18.86 \%, 32.56 \%]$} & {$[0.22 \%, 0.81 \%]$} \\
& HTC & & {$[34.18 \%, 82.60 \%]$} & {$[0.45 \%, 1.14 \%]$} \\
\hline
\end{tabular}


Table 17: Counterfactual Result, Robustness Check 5

\begin{tabular}{ccccc}
\hline & & No VI & Investment Coordination & Full VI \\
\hline \hline & Apple & {$[0.0405,0.0407]$} & {$[0.0410,0.0412]$} & {$[0.0410,0.0411]$} \\
& Samsung & {$[0.1030,0.1070]$} & {$[0.1149,0.1178]$} & {$[0.1155,0.1180]$} \\
& HTC & {$[0.0745,0.0854]$} & {$[0.0905,0.0967]$} & {$[0.0909,0.0969]$} \\
Innovation Rate: & Qualcomm & {$[0.0677,0.0736]$} & {$[0.0824,0.0851]$} & {$[0.0831,0.0859]$} \\
\cline { 2 - 5 }$\left(q_{36}-q_{1}\right) / 35$ & & Difference \% & \\
\cline { 2 - 5 } & Apple & {$[1.14 \%, 1.28 \%]$} & {$[-0.21 \%, 0.00 \%]$} \\
& Samsung & {$[9.57 \%, 14.29 \%]$} & {$[0.22 \%, 0.54 \%]$} \\
& HTC & {$[13.28 \%, 21.40 \%]$} & {$[0.15 \%, 0.51 \%]$} \\
& Qualcomm & {$[15.65 \%, 24.21 \%]$} & {$[0.55 \%, 0.88 \%]$} \\
\hline
\end{tabular}

Table 18: Counterfactual Result, Robustness Check 6

\begin{tabular}{ccccc}
\hline & & No VI & Investment Coordination & Full VI \\
\hline \hline & Apple & {$[0.0380,0.0443]$} & {$[0.0386,0.0442]$} & {$[0.0385,0.0442]$} \\
& Samsung & {$[0.0877,0.1054]$} & {$[0.1352,0.1436]$} & {$[0.1359,0.1441]$} \\
& HTC & {$[0.0771,0.0971]$} & {$[0.1067,0.1307]$} & {$[0.1068,0.1309]$} \\
Innovation Rate: & Qualcomm & {$[0.0752,0.0910]$} & {$[0.1299,0.1358]$} & {$[0.1308,0.1367]$} \\
\cline { 2 - 5 }$\left(q_{36}-q_{1}\right) / 35$ & & & Difference \% & \\
\cline { 2 - 5 } & Apple & {$[-0.34 \%, 1.68 \%]$} & {$[-0.45 \%, 0.00 \%]$} \\
& Samsung & {$[36.28 \%, 55.74 \%]$} & {$[0.30 \%, 0.53 \%]$} \\
& HTC & & {$[34.61 \%, 41.07 \%]$} & {$[0.14 \%, 0.62 \%]$} \\
& Qualcomm & {$[46.73 \%, 74.50 \%]$} & {$[0.37 \%, 0.70 \%]$} \\
\hline
\end{tabular}

Table 19: Counterfactual Result, Robustness Check 7

\begin{tabular}{ccccc}
\hline & & No VI & Investment Coordination & Full VI \\
\hline \hline & Apple & {$[0.0401,0.0477]$} & {$[0.0404,0.0476]$} & {$[0.0404,0.0475]$} \\
& Samsung & {$[0.0895,0.1054]$} & {$[0.1188,0.1474]$} & {$[0.1198,0.1481]$} \\
& HTC & {$[0.0566,0.0778]$} & {$[0.0667,0.1062]$} & {$[0.0667,0.1066]$} \\
Innovation Rate: & Qualcomm & {$[0.0906,0.1067]$} & {$[0.1200,0.1510]$} & {$[0.1209,0.1518]$} \\
\cline { 2 - 5 }$\left(q_{36}-q_{1}\right) / 35$ & & Difference \% & \\
\cline { 2 - 5 } & Apple & {$[-0.36 \%, 0.85 \%]$} & {$[-0.07 \%, 0.00 \%]$} \\
& Samsung & {$[27.74 \%, 58.66 \%]$} & {$[0.43 \%, 0.83 \%]$} \\
& HTC & & {$[17.73 \%, 42.44 \%]$} & {$[0.04 \%, 0.30 \%]$} \\
& Qualcomm & {$[26.20 \%, 61.58 \%]$} & {$[0.51 \%, 0.78 \%]$} \\
\hline
\end{tabular}


Table 20: Vertical Integration with Potential Entry. Innovation Rate: $\left(q_{36}-q_{1}\right) / 35$

\begin{tabular}{ccc}
\hline & No VI & VI \\
& $\bar{\psi}$ & $0.9 \bar{\psi}$ \\
\hline \hline Apple & {$[0.0359,0.0372]$} & {$[0.0414,0.0454]$} \\
Samsung & {$[0.0979,0.1064]$} & {$[0.1314,0.1288]$} \\
HTC & {$[0.0666,0.0674]$} & {$[0.0939,0.0953]$} \\
Qualcomm & {$[0.0638,0.0737]$} & {$[0.0979,0.0993]$} \\
\hline
\end{tabular}

